
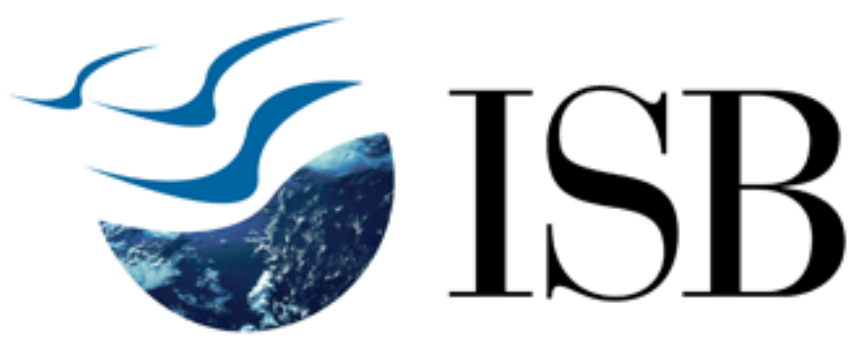

A Duality-Based Relaxation and Decomposition Approach for Inventory

Distribution Systems

http://eprints.exchange.isb.edu/98/

Article

Indian School of Business

2008 


\title{
A Duality-Based Relaxation and Decomposition Approach for Inventory Distribution Systems
}

\author{
Sumit Kunnumkal, ${ }^{1}$ Huseyin Topaloglu ${ }^{2}$ \\ ${ }^{1}$ Indian School of Business, Gachibowli, Hyderabad 500032, India \\ ${ }^{2}$ School of Operations Research and Information Engineering, Cornell University, Ithaca, New York 14853
}

Received 20 February 2007; revised 18 May 2008; accepted 24 May 2008

DOI 10.1002/nav.20306

Published online 25 August 2008 in Wiley InterScience (www.interscience.wiley.com).

\begin{abstract}
We propose a new method for making the inventory replenishment decisions in distribution systems. In particular, we consider distribution systems consisting of multiple retailers that face random demand and a warehouse that supplies the retailers. The method that we propose is based on formulating the distribution problem as a dynamic program, and relaxing the constraints that ensure the nonnegativity of the shipments to the retailers, by associating Lagrange multipliers with them. We show that our method provides lower bounds on the value functions, and a good set of values for the Lagrange multipliers can be obtained by maximizing a concave function in a relatively straightforward manner. Computational experiments indicate that our method can provide significant improvements over the traditional approaches for making the inventory replenishment decisions, in terms of both the tightness of the lower bounds on the value functions and the performance of the policies. (c) 2008 Wiley Periodicals, Inc. Naval Research Logistics 55: 612-631, 2008
\end{abstract}

Keywords: inventory distribution; balance assumption; Lagrangian relaxation

\section{INTRODUCTION}

This work proposes a new method for making the inventory replenishment decisions in a distribution system consisting of multiple retailers and a warehouse. The retailers face random demand and the demand that cannot be met is backlogged. The warehouse supplies the retailers and replenishes itself from an external supplier. We are interested in finding a policy to supply the retailers and to replenish the warehouse so as to minimize the total expected cost over a finite-planning horizon.

Two standard approaches for making the inventory replenishment decisions in distribution systems are due to Clark and Scarf [8] and Federgruen and Zipkin [14]. In particular, the seminal paper by Clark and Scarf [8] introduces the balance assumption, which amounts to assuming that it is never desirable to redistribute the total amount of retailer inventory among the retailers even if it is allowed to do so. Under the balance assumption, it is possible to show that the optimal inventory replenishment policy can be found by focusing on one installation at a time. Federgruen and

Correspondence to: H. Topaloglu (topaloglu@ orie.cornell.edu)
Zipkin [14] propose relaxing the requirement that the shipments to the retailers are non-negative. Under the assumption that negative shipments to the retailers are allowed, it is also possible to show that the optimal inventory replenishment policy can be found by focusing on one installation at a time. Because this assumption is a relaxation on the original problem, their relaxation strategy obtains lower bounds on the value functions. There has been much computational work showing that the inventory replenishment policies obtained under the balance assumption of Clark and Scarf [8] and the relaxation strategy of Federgruen and Zipkin [14] perform quite well. Nevertheless, the comprehensive computational work by Dogru [9], among others, indicates that there are still many practically important settings where the balance assumption and the relaxation strategy remain inadequate. We shortly list some of these settings. This work proposes a viable alternative when such inadequacies arise.

The method that we propose in this work is based on formulating the distribution problem as a dynamic program and relaxing the constraints that ensure the nonnegativity of the shipments to the retailers. Although a similar idea is used by the relaxation strategy of Federgruen and Zipkin [14], the novel aspect of our method is that it explicitly associates Lagrange multipliers with the relaxed constraints, 
whereas Federgruen and Zipkin [14] simply drop the constraints without using any penalty terms. It turns out that the presence of the Lagrange multipliers significantly improves the lower bounds on the value functions and the performance of the policies. Furthermore, we show that a good set of values for the Lagrange multipliers can be obtained by maximizing a concave function in a relatively straightforward manner. Computational experiments indicate that although our method does not always provide better performance than the inventory replenishment policies obtained under the balance assumption of Clark and Scarf [8], it can perform well when the balance assumption remains inadequate. Furthermore, the relaxation strategy of Federgruen and Zipkin [14] can be obtained simply by setting all the Lagrange multipliers in our method to zero, whereas our method tightens the lower bounds on the value functions by adjusting the Lagrange multipliers over a set that includes zero. As a result, our method naturally improves the lower bounds on the value functions that are computed under the relaxation strategy of Federgruen and Zipkin [14].

There is extensive literature on distribution systems. The balance assumption introduced by Clark and Scarf [8] plays a crucial role in this area, and it recurrently appears in many settings. Eppen and Schrage [11] consider a distribution system consisting of identical retailers that face normally distributed demand and a warehouse that does not hold inventory. They derive closed-form expressions for the inventory control parameters by using the balance assumption. Jackson [18] extends this work to allow holding inventory at the warehouse. Axsater et al. [3] uses the balance assumption for a distribution system where the replenishment orders of the warehouse have to be in multiples of a given batch quantity. Dogru [9] and Dogru et al. [10] give comprehensive computational studies that test the validity of the balance assumption. Axsater [2] provides a recent review of the literature revolving around the balance assumption. On the other hand, the idea of relaxing the constraints that ensure the non-negativity of the shipments to the retailers first appears in Federgruen and Zipkin [13, 14]. These articles consider distribution systems with stockless warehouses, nonidentical retailers, finite-planning horizons, and nonstationary demand distributions. Federgruen and Zipkin [15] revisit the relaxation strategy when the planning horizon is infinite, and they obtain closed-form expressions for the inventory control parameters. Federgruen [12] provides a review of the applications of the relaxation strategy on both serial and distribution systems. Gallego et al. [17] compare the performance of the relaxation strategy with several heuristics. In general, the inventory replenishment policies obtained under the balance assumption of Clark and Scarf [8] and the relaxation strategy of Federgruen and Zipkin [14] perform quite well. Nevertheless, the computational experiments in Refs. $[9,10,13]$ consistently indicate that these approaches may not be satisfactory when the coefficient of variation for the demand random variables is high, the backlogging costs at the retailers are high, and there are large differences in the cost parameters of different installations.

The idea of relaxing the constraints in a dynamic program by associating Lagrange multipliers with them appears in the literature, and the connection between duality theory and control theory is clearly documented in Refs. [6,23]. Adelman and Mersereau [1] recently revive interest in this connection by studying dynamic programs that would decompose by the components of the state variable if a few constraints did not link the different components of the state variable. They use the term "weakly coupled" to refer such dynamic programs. Karmarkar, Cheung and Powell, Topaloglu and Kunnumkal, and Topaloglu [7, 19-22] use the weakly coupled dynamic programming framework in multilocation inventory control, dynamic fleet management, and network revenue management settings.

The main research contribution of this work is to propose a new method for making the inventory replenishment decisions in a distribution system. Our method formulates the distribution problem as a dynamic program and relaxes certain constraints by associating Lagrange multipliers with them. We show that our method provides lower bounds on the value functions, and a good set of values for the Lagrange multipliers can be obtained by maximizing a concave function. Because the relaxation strategy of Federgruen and Zipkin [14] is a special case of our method that is obtained by setting all the Lagrange multipliers to zero, we naturally improve the lower bounds on the value functions that are computed under their relaxation strategy. From computational standpoint, we demonstrate that the lower bounds on the value functions and the performance of the policies obtained by our method can be significantly better than those obtained under the balance assumption of Clark and Scarf [8] and the relaxation strategy of Federgruen and Zipkin [14]. We empirically identify the conditions under which the policies obtained by our method perform better than the policies obtained under the balance assumption of Clark and Scarf [8] and the relaxation strategy of Federgruen and Zipkin [14].

The rest of the article is organized as follows. Section 2 formulates the distribution problem as a dynamic program. Section 3 describes our Lagrangian relaxation strategy. Sections 4 and 5, respectively, review the balance assumption of Clark and Scarf [8] and the relaxation strategy of Federgruen and Zipkin [14]. We note that we refer to our method as the Lagrangian relaxation strategy because our method explicitly associates Lagrange multipliers with the relaxed constraints, whereas we refer to the method proposed by Federgruen and Zipkin [14] simply as the relaxation strategy. Section 6 compares the balance assumption of Clark and Scarf [8] and the relaxation strategy of Federgruen and Zipkin [14] with our method. Section 7 shows that applying 
the inventory replenishment policies obtained by our method requires solving simple optimization problems with separable piecewise-linear convex objective functions. Section 8 presents computational experiments.

\section{PROBLEM FORMULATION}

We consider a distribution system consisting of multiple retailers and a warehouse. The retailers face random demand, and they are supplied by the warehouse. The warehouse is supplied by an external supplier with infinite supply. The problem takes place over the finite-planning horizon $\mathcal{I}=\{1, \ldots, \tau\}$. The set of retailers is $\mathcal{I}$, and we denote the warehouse by $\phi$. We use the term installation when we want to refer to a retailer or the warehouse without making a distinction. We let $D_{i t}$ be the demand at retailer $i$ at time period $t$. We assume that the demands at different retailers or at different time periods are independent. We let $D_{\phi t}=\sum_{i \in \mathcal{I}} D_{i t}$ so that we can also refer to the demand at the warehouse.

For notational clarity, we assume that the lead times for all replenishments are zero. In other words, the replenishment order shipped to a certain installation at a certain time period reaches the installation at the same time period. Using standard arguments, one can show that all our results extend in a tractable manner to cover the case where the lead times are nonzero. In particular, one can use the on-hand inventory, plus the total pipeline inventory, minus the backlogs as the inventory position at an installation, and it is not necessary to keep the detailed profile of the pipeline inventory by using an extended state variable. The computational results that we present in Section 8 indeed consider test problems with nonzero lead times. Again, for notational clarity, we only consider distribution systems consisting of a warehouse serving multiple retailers, but our results extend to distribution systems with multiple distribution stages through the same argument in Section 3.3.1 in Federgruen [12]. Under the assumption that the lead times are zero and there is one distribution stage, the following sequence of events takes place at a particular time period. (1) The warehouse places its replenishment order from the external supplier. (2) Based on its product availability and the inventory position at the retailers, the warehouse supplies the retailers. (3) The retailers and the warehouse receive the replenishment orders that were shipped at the current time period. (4) The demands at the retailers are observed. The unsatisfied demands at the retailers are backlogged. The holding and backlogging costs are incurred.

We let $x_{i t}$ be the echelon inventory position at installation $i$ at the beginning of time period $t$. For retailer $i$, because the lead times are zero, $x_{i t}$ corresponds to the on-hand inventory minus the backlogs at the retailer. For the warehouse, $x_{\phi t}$ corresponds to the on-hand inventory at the warehouse, plus the on-hand inventory at all of the retailers, minus the backlogs at all of the retailers. Therefore, $x_{\phi t}-\sum_{i \in \mathcal{I}} x_{i t}$ is the on-hand inventory at the warehouse at the beginning of time period $t$. We refer the reader to Clark and Scarf [8] and Federgruen [12] for a detailed discussion of the echelon inventory concept. We let $q_{i t}$ be the replenishment order shipped to installation $i$ at time period $t$. Because the replenishment orders are received after the warehouse supplies the retailers and before the demands at the retailers are observed, the warehouse has $x_{\phi t}-\sum_{i \in \mathcal{I}} x_{i t}$ units of product to supply the retailers and retailer $i$ has $x_{i t}+q_{i t}$ units of product to serve the demand at time period $t$.

We let $h_{i t}$ be the per unit holding cost at installation $i$ at time period $t$ and $b_{i t}$ be the per unit backlogging cost at retailer $i$ at time period $t$. In this case, the expected one-period holding and backlogging cost incurred at time period $t$ by the whole distribution system can be written as

$$
\begin{aligned}
& h_{\phi t}\left[x_{\phi t}-\sum_{i \in \mathcal{I}} x_{i t}+q_{\phi t}-\sum_{i \in \mathcal{I}} q_{i t}\right] \\
& +\sum_{i \in \mathcal{I}} h_{i t} \mathbb{E}\left\{\left[x_{i t}+q_{i t}-D_{i t}\right]^{+}\right\}+\sum_{i \in \mathcal{I}} b_{i t} \mathbb{E}\left\{\left[D_{i t}-x_{i t}-q_{i t}\right]^{+}\right\},
\end{aligned}
$$

where we use $[\cdot]^{+}=\max \{\cdot, 0\}$. The warehouse does not face the customer demand, and there is no backlogging cost at this installation. If we let $L_{\phi t}\left(x_{\phi t}+q_{\phi t}\right)=h_{\phi t}\left[x_{\phi t}+q_{\phi t}\right]$ and

$$
\begin{aligned}
L_{i t}\left(x_{i t}+q_{i t}\right)=-h_{\phi t}\left[x_{i t}+q_{i t}\right] & +h_{i t} \mathbb{E}\left\{\left[x_{i t}+q_{i t}-D_{i t}\right]^{+}\right\} \\
& +b_{i t} \mathbb{E}\left\{\left[D_{i t}-x_{i t}-q_{i t}\right]^{+}\right\},
\end{aligned}
$$

then we can write the expected one-period holding and backlogging cost incurred at time period $t$ by the whole distribution system as $\sum_{i \in \mathcal{I} \cup\{\phi\}} L_{i t}\left(x_{i t}+q_{i t}\right)$. This is a standard way of accounting for the expected one-period holding and backlogging costs in the literature; see Federgruen [12].

Using $x_{t}=\left\{x_{i t}: i \in \mathcal{I} \cup\{\phi\}\right\}$ as the state variable at time period $t$, and letting $q_{t}=\left\{q_{i t}: i \in \mathcal{I} \cup\{\phi\}\right\}$ be the vector of shipment quantities to the installations and $D_{t}=\left\{D_{i t}: i \in \mathcal{I} \cup\{\phi\}\right\}$ be the vector of demands at time period $t$, the optimal policy that minimizes the total expected cost can be found by computing the value functions $\left\{V_{t}(\cdot): t \in \mathcal{T}\right\}$ through the optimality equation

$$
\begin{aligned}
V_{t}\left(x_{t}\right)=\min & \sum_{i \in \mathcal{I} \cup\{\phi\}} c_{i t} q_{i t}+\sum_{i \in \mathcal{I} \cup\{\phi\}} L_{i t}\left(x_{i t}+q_{i t}\right) \\
& +\mathbb{E}\left\{V_{t+1}\left(x_{t}+q_{t}-D_{t}\right)\right\} \\
\text { subject to } & \sum_{i \in \mathcal{I}} q_{i t} \leq x_{\phi t}-\sum_{i \in \mathcal{I}} x_{i t} \\
& q_{i t} \geq 0 \quad \text { for all } i \in \mathcal{I} \cup\{\phi\},
\end{aligned}
$$

where $c_{i t}$ is the per unit replenishment cost at installation $i$ at time period $t$. Because $x_{\phi t}$ includes the on-hand inventory 
and backlogs at the retailers, the echelon inventory position at the warehouse at time period $t+1$ is computed as $x_{\phi t}+q_{\phi t}-D_{\phi t}$. The first constraint in the problem above ensures that the shipments to the retailers do not violate the inventory availability at the warehouse. Defining the decision variables $y_{t}=\left\{y_{i t}: i \in \mathcal{I} \cup\{\phi\}\right\}$ as $y_{i t}=x_{i t}+q_{i t}$, the optimality equation above becomes

$$
\begin{aligned}
V_{t}\left(x_{t}\right)=\min & \sum_{i \in \mathcal{I} \cup\{\phi\}} c_{i t}\left[y_{i t}-x_{i t}\right]+\sum_{i \in \mathcal{I} \cup\{\phi\}} L_{i t}\left(y_{i t}\right) \\
& +\mathbb{E}\left\{V_{t+1}\left(y_{t}-D_{t}\right)\right\} \\
\text { subject to } & \sum_{i \in \mathcal{I}} y_{i t} \leq x_{\phi t} \\
& y_{i t} \geq x_{i t} \text { for all } i \in \mathcal{I} \cup\{\phi\}
\end{aligned}
$$

Because of the large number of dimensions of the state variable, solving the optimality equation above through classical dynamic programming techniques is difficult. In the next section, we propose a Lagrangian relaxation strategy that relaxes the constraints $y_{i t} \geq x_{i t}$ for all $i \in \mathcal{I}$ in problem (1)-(3) by associating positive Lagrange multipliers with them. In this case, the optimality equation decomposes by the installations. We make this idea precise in the next section.

\section{LAGRANGIAN RELAXATION STRATEGY}

Associating the positive Lagrange multipliers $\lambda=\left\{\lambda_{i t}\right.$ : $i \in \mathcal{I}, t \in \mathcal{T}\}$ with the constraints $y_{i t} \geq x_{i t}$ for all $i \in \mathcal{I}$ in problem (1)-(3), the Lagrangian relaxation strategy solves the optimality equation

$$
\begin{aligned}
V_{t}^{L}\left(x_{t} \mid \lambda\right)=\min & c_{\phi t}\left[y_{\phi_{t}}-x_{\phi t}\right]+L_{\phi t}\left(y_{\phi t}\right) \\
& +\sum_{i \in \mathcal{I}}\left[c_{i t}-\lambda_{i t}\right]\left[y_{i t}-x_{i t}\right] \\
& +\sum_{i \in \mathcal{I}} L_{i t}\left(y_{i t}\right)+\mathbb{E}\left\{V_{t+1}^{L}\left(y_{t}-D_{t} \mid \lambda\right)\right\}
\end{aligned}
$$

$$
\begin{array}{ll}
\text { subject to } & \sum_{i \in \mathcal{I}} y_{i t} \leq x_{\phi t} \\
& y_{\phi t} \geq x_{\phi t},
\end{array}
$$

where the argument $\lambda$ in the value functions emphasizes that the solution to the optimality equation above depends on the Lagrange multipliers. Because we have $q_{i t}=y_{i t}-x_{i t}$, relaxing the constraints $y_{i t} \geq x_{i t}$ for all $i \in \mathcal{I}$ is equivalent to relaxing the constraints that ensure the non-negativity of the shipments to the retailers. We also note that the Lagrange multipliers $\left\{\lambda_{i t}: i \in \mathcal{I}\right\}$ in problem (4)-(6) do not depend on the state variable $x_{t}$.
The material in this section is divided between two subsections. Section 3.1 shows that for given Lagrange multipliers, the optimality equation in (4)-(6) decomposes by the installations. This is the main computational advantage of the Lagrangian relaxation strategy. Section 3.2, on the other hand, shows that the value functions $\left\{V_{t}^{L}(\cdot \mid \lambda): t \in \mathcal{T}\right\}$ computed through the optimality equation in (4)-(6) provide lower bounds on the value functions $\left\{V_{t}(\cdot): t \in \mathcal{T}\right\}$ computed through the optimality equation in (1)-(3). In addition, Section 3.2 resolves the question of how to choose a good set of values for the Lagrange multipliers. Once we are able to choose a good set of values for the Lagrange multipliers, we can use $\left\{V_{t}^{L}(\cdot \mid \lambda): t \in \mathcal{T}\right\}$ as approximations to $\left\{V_{t}(\cdot): t \in \mathcal{T}\right\}$. In particular, we can replace $\left\{V_{t}(\cdot): t \in \mathcal{T}\right\}$ in problem (1)-(3) with $\left\{V_{t}^{L}(\cdot \mid \lambda): t \in \mathcal{T}\right\}$ and solve this problem to make the inventory replenishment decisions.

\subsection{Solving the Optimality Equation under the Lagrangian Relaxation Strategy}

Our main result in this section shows that for given Lagrange multipliers, the value functions computed under the Lagrangian relaxation strategy are separable functions of the form

$$
V_{t}^{L}\left(x_{t} \mid \lambda\right)=\sum_{i \in \mathcal{I} \cup\{\phi\}} v_{i t}^{L}\left(x_{i t} \mid \lambda\right) .
$$

In the expression above, the value functions $\left\{v_{i t}^{L}(\cdot \mid \lambda): t \in \mathcal{T}\right\}$ for retailer $i$ are computed through the optimality equation

$$
\begin{gathered}
v_{i t}^{L}\left(x_{i t} \mid \lambda\right)=\min _{y_{i t}} \quad\left\{\left[c_{i t}-\lambda_{i t}\right]\left[y_{i t}-x_{i t}\right]+L_{i t}\left(y_{i t}\right)\right. \\
\left.+\mathbb{E}\left\{v_{i, t+1}^{L}\left(y_{i t}-D_{i t} \mid \lambda\right)\right\}\right\} .
\end{gathered}
$$

This optimality equation computes the optimal inventory replenishment policy for retailer $i$ under the assumption that the warehouse has infinite supply, the replenishment quantities of retailer $i$ are not restricted to be positive, and the per unit replenishment costs at retailer $i$ are deflated by $\left\{\lambda_{i t}: t \in \mathcal{T}\right\}$. On the other hand, noting that the optimal solution to problem (8) does not depend on $x_{i t}$ and letting $\hat{r}_{i t}^{\lambda}$ be the optimal solution to this problem, the value functions $\left\{v_{\phi t}^{L}(\cdot \mid \lambda): t \in \mathcal{T}\right\}$ for the warehouse are computed through the optimality equation

$$
\begin{aligned}
v_{\phi t}^{L}\left(x_{\phi t} \mid \lambda\right)=\min _{y_{\phi t} \geq x_{\phi t}} & \left\{c_{\phi t}\left[y_{\phi t}-x_{\phi t}\right]+L_{\phi t}\left(y_{\phi t}\right)\right. \\
& \left.+\mathbb{E}\left\{v_{\phi, t+1}^{L}\left(y_{\phi t}-D_{\phi t} \mid \lambda\right)\right\}\right\} \\
& +\Delta_{t}^{L}\left(x_{\phi t} \mid \lambda\right),
\end{aligned}
$$


where the functions $\left\{\Delta_{t}^{L}(\cdot \mid \lambda): t \in \mathcal{T}\right\}$ are given by

$$
\begin{aligned}
\Delta_{t}^{L}\left(x_{\phi t} \mid \lambda\right)=\min \quad & \sum_{i \in \mathcal{I}}\left[c_{i t}-\lambda_{i t}\right]\left[y_{i t}-\hat{r}_{i t}^{\lambda}\right]+\sum_{i \in \mathcal{I}} L_{i t}\left(y_{i t}\right) \\
& +\sum_{i \in \mathcal{I}} \mathbb{E}\left\{v_{i, t+1}^{L}\left(y_{i t}-D_{i t} \mid \lambda\right)\right\} \\
& -\sum_{i \in \mathcal{I}} L_{i t}\left(\hat{r}_{i t}^{\lambda}\right) \\
& -\sum_{i \in \mathcal{I}} \mathbb{E}\left\{v_{i, t+1}^{L}\left(\hat{r}_{i t}^{\lambda}-D_{i t} \mid \lambda\right)\right\} \\
\text { subject to } & \sum_{i \in \mathcal{I}} y_{i t} \leq x_{\phi t} .
\end{aligned}
$$

We note that the optimality equation in (9) computes the optimal inventory replenishment policy for the warehouse under the assumption that the expected one-period costs at the warehouse are inflated by using the functions $\left\{\Delta_{t}^{L}(\cdot \mid \lambda)\right.$ : $t \in \mathcal{T}\}$.

The next proposition shows that the value functions computed under the Lagrangian relaxation strategy are separable functions of the form (7). The fact that the optimal solution to problem (8) does not depend on $x_{i t}$ plays an important role in this result.

PROPOSITION 1: If the value functions $\left\{V_{t}^{L}(\cdot \mid \lambda): t \in\right.$ $\mathcal{T}\},\left\{v_{i t}^{L}(\cdot \mid \lambda): i \in \mathcal{I}, t \in \mathcal{T}\right\}$, and $\left\{v_{\phi t}^{L}(\cdot \mid \lambda): t \in \mathcal{T}\right\}$ are respectively computed through the optimality equations in (4)-(6), (8), and (9), then we have

$$
V_{t}^{L}\left(x_{t} \mid \lambda\right)=\sum_{i \in \mathcal{I} \cup\{\phi\}} v_{i t}^{L}\left(x_{i t} \mid \lambda\right)
$$

for all $t \in \mathcal{T}$.

PROOF: We show the result by induction over the time periods. It is easy to show the result for the last time period. Assuming that the result holds for time period $t+1$, problem (4)-(6) imply that

$$
\begin{aligned}
V_{t}^{L}\left(x_{t} \mid \lambda\right)=\min & c_{\phi t}\left[y_{\phi_{t}}-x_{\phi t}\right]+L_{\phi t}\left(y_{\phi t}\right) \\
& +\mathbb{E}\left\{v_{\phi, t+1}^{L}\left(y_{\phi t}-D_{\phi t} \mid \lambda\right)\right\} \\
& +\sum_{i \in \mathcal{I}}\left[c_{i t}-\lambda_{i t}\right]\left[y_{i t}-x_{i t}\right]+\sum_{i \in \mathcal{I}} L_{i t}\left(y_{i t}\right) \\
& +\sum_{i \in \mathcal{I}} \mathbb{E}\left\{v_{i, t+1}^{L}\left(y_{i t}-D_{i t} \mid \lambda\right)\right\}
\end{aligned}
$$

subject to (5), (6).

Because $\hat{r}_{i t}^{\lambda}$ is the optimal solution to problem (8), we have

$$
\begin{aligned}
v_{i t}^{L}\left(x_{i t} \mid \lambda\right)=\left[c_{i t}-\lambda_{i t}\right]\left[\hat{r}_{i t}^{\lambda}-x_{i t}\right]+ & L_{i t}\left(\hat{r}_{i t}^{\lambda}\right) \\
& +\mathbb{E}\left\{v_{i, t+1}^{L}\left(\hat{r}_{i t}^{\lambda}-D_{i t} \mid \lambda\right)\right\} .
\end{aligned}
$$

Adding and subtracting $\sum_{i \in \mathcal{I}} v_{i t}^{L}\left(x_{i t} \mid \lambda\right)$ and using the expression above, we can write the objective function of problem (12)-(13) as

$$
\begin{aligned}
c_{\phi t}\left[y_{\phi_{t}}-\right. & \left.x_{\phi t}\right]+L_{\phi t}\left(y_{\phi t}\right)+\mathbb{E}\left\{v_{\phi, t+1}^{L}\left(y_{\phi t}-D_{\phi t} \mid \lambda\right)\right\} \\
& +\sum_{i \in \mathcal{I}} v_{i t}^{L}\left(x_{i t} \mid \lambda\right)+\sum_{i \in \mathcal{I}}\left[c_{i t}-\lambda_{i t}\right]\left[y_{i t}-\hat{r}_{i t}^{\lambda}\right] \\
+ & \sum_{i \in \mathcal{I}} L_{i t}\left(y_{i t}\right)+\sum_{i \in \mathcal{I}} \mathbb{E}\left\{v_{i, t+1}^{L}\left(y_{i t}-D_{i t} \mid \lambda\right)\right\} \\
& -\sum_{i \in \mathcal{I}} L_{i t}\left(\hat{r}_{i t}^{\lambda}\right)-\sum_{i \in \mathcal{I}} \mathbb{E}\left\{v_{i, t+1}^{L}\left(\hat{r}_{i t}^{\lambda}-D_{i t} \mid \lambda\right)\right\} .
\end{aligned}
$$

The decision variables $\left\{y_{i t}: i \in \mathcal{I}\right\}$ appear only in constraints (5), whereas the decision variable $y_{\phi t}$ appears only in constraint (6) in problem (12)-(13). Therefore, using (14) and the definition of $\Delta_{t}^{L}\left(x_{\phi t} \mid \lambda\right)$ in problem (10)-(11), problem (12)-(13) become

$$
\begin{aligned}
V_{t}^{L}\left(x_{t} \mid \lambda\right)=\min _{y_{\phi t} \geq x_{\phi t}} & \left\{c_{\phi t}\left[y_{\phi_{t}}-x_{\phi t}\right]+L_{\phi t}\left(y_{\phi t}\right)\right. \\
& \left.+\mathbb{E}\left\{v_{\phi, t+1}^{L}\left(y_{\phi t}-D_{\phi t} \mid \lambda\right)\right\}\right\} \\
& +\sum_{i \in \mathcal{I}} v_{i t}^{L}\left(x_{i t} \mid \lambda\right)+\Delta_{t}^{L}\left(x_{\phi t} \mid \lambda\right),
\end{aligned}
$$

in which case (9) implies that the result holds for time period $t$.

The practical significance of the Lagrangian relaxation strategy is that for given Lagrange multipliers, the optimality equation in (4)-(6) can be solved by focusing on one installation at a time. In particular, we first solve the optimality equation in (8) to compute $\left\{v_{i t}^{L}(\cdot \mid \lambda): i \in \mathcal{I}, t \in \mathcal{T}\right\}$. In this case, we can compute $\left\{\Delta_{t}^{L}(\cdot \mid \lambda): t \in \mathcal{T}\right\}$ by solving problem (10)-(11). Finally, we can compute $\left\{v_{\phi t}^{L}(\cdot \mid \lambda): t \in \mathcal{T}\right\}$ by solving the optimality equation in (9).

The next lemma shows that the optimality equation in (8) can be solved myopically for given Lagrange multipliers. This result further enhances the computational tractability of the Lagrangian relaxation strategy.

LEMMA 2: Using the boundary condition that $c_{i, \tau+1}=$ $\lambda_{i, \tau+1}=0$ and noting that the optimal solution to problem (8) is $\hat{r}_{i t}^{\lambda}$, the optimal solution to the problem

$$
\min _{y_{i t}}\left\{\left\{\left[c_{i t}-\lambda_{i t}\right]-\left[c_{i, t+1}-\lambda_{i, t+1}\right]\right\}\left[y_{i t}-x_{i t}\right]+L_{i t}\left(y_{i t}\right)\right\}
$$


is also $\hat{r}_{i t}^{\lambda}$. Furthermore, we have

$$
\begin{array}{r}
v_{i t}^{L}\left(x_{i t} \mid \lambda\right)=\left[c_{i t}-\lambda_{i t}\right]\left[\hat{r}_{i t}^{\lambda}-x_{i t}\right]+L_{i t}\left(\hat{r}_{i t}^{\lambda}\right) \\
+\sum_{t^{\prime}=t+1}^{\tau}\left[c_{i t^{\prime}}-\lambda_{i t^{\prime}}\right]\left[\hat{r}_{i t^{\prime}}^{\lambda}-\hat{r}_{i, t^{\prime}-1}^{\lambda}+\mathbb{E}\left\{D_{i, t^{\prime}-1}\right\}\right] \\
+\sum_{t^{\prime}=t+1}^{\tau} L_{i t^{\prime}}\left(\hat{r}_{i t^{\prime}}^{\lambda}\right) .
\end{array}
$$

PROOF: We show the result by induction over the time periods. It is easy to show the result for the last time period. Assuming that the result holds for time period $t+1$, we have

$$
\begin{aligned}
& \hat{r}_{i t}^{\lambda}=\underset{y_{i t}}{\operatorname{argmin}}\left\{\left[c_{i t}-\lambda_{i t}\right]\left[y_{i t}-x_{i t}\right]+L_{i t}\left(y_{i t}\right)\right. \\
& \left.+\mathbb{E}\left\{v_{i, t+1}^{L}\left(y_{i t}-D_{i t} \mid \lambda\right)\right\}\right\} \\
& =\underset{y_{i t}}{\operatorname{argmin}}\left\{\left[c_{i t}-\lambda_{i t}\right]\left[y_{i t}-x_{i t}\right]+L_{i t}\left(y_{i t}\right)\right. \\
& \left.+\left[c_{i, t+1}-\lambda_{i, t+1}\right] \mathbb{E}\left\{\hat{r}_{i, t+1}^{\lambda}-y_{i t}+D_{i t}\right\}\right\} \\
& +L_{i, t+1}\left(\hat{r}_{i, t+1}^{\lambda}\right)+\sum_{t^{\prime}=t+2}^{\tau}\left[c_{i t^{\prime}}-\lambda_{i t^{\prime}}\right]\left[\hat{r}_{i t^{\prime}}^{\lambda}-\hat{r}_{i, t^{\prime}-1}^{\lambda}\right. \\
& \left.+\mathbb{E}\left\{D_{i, t^{\prime}-1}\right\}\right]+\sum_{t^{\prime}=t+2}^{\tau} L_{i t^{\prime}}\left(\hat{r}_{i t^{\prime}}^{\lambda}\right) \\
& =\underset{y_{i t}}{\operatorname{argmin}}\left\{\left\{\left[c_{i t}-\lambda_{i t}\right]-\left[c_{i, t+1}-\lambda_{i, t+1}\right]\right\}\left[y_{i t}-x_{i t}\right]\right. \\
& \left.+L_{i t}\left(y_{i t}\right)\right\}
\end{aligned}
$$

which shows that the optimal solution to problem (15) is $\hat{r}_{i t}^{\lambda}$. In this case, the fact that $\hat{r}_{i t}^{\lambda}$ is the optimal solution to problem (8) and the induction assumption implies that

$$
\begin{aligned}
v_{i t}^{L}\left(x_{i t} \mid \lambda\right)= & {\left[c_{i t}-\lambda_{i t}\right]\left[\hat{r}_{i t}^{\lambda}-x_{i t}\right]+L_{i t}\left(\hat{r}_{i t}^{\lambda}\right) } \\
& +\mathbb{E}\left\{v_{i, t+1}^{L}\left(\hat{r}_{i t}^{\lambda}-D_{i t} \mid \lambda\right)\right\} \\
= & {\left[c_{i t}-\lambda_{i t}\right]\left[\hat{r}_{i t}^{\lambda}-x_{i t}\right]+L_{i t}\left(\hat{r}_{i t}^{\lambda}\right)+\left[c_{i, t+1}-\lambda_{i, t+1}\right] } \\
& \times \mathbb{E}\left\{\hat{r}_{i, t+1}^{\lambda}-\hat{r}_{i t}^{\lambda}+D_{i t}\right\} \\
& +L_{i, t+1}\left(\hat{r}_{i, t+1}^{\lambda}\right)+\sum_{t^{\prime}=t+2}^{\tau}\left[c_{i t^{\prime}}-\lambda_{i t^{\prime}}\right] \\
& \times\left[\hat{r}_{i t^{\prime}}^{\lambda}-\hat{r}_{i, t^{\prime}-1}^{\lambda}+\mathbb{E}\left\{D_{i, t^{\prime}-1}\right\}\right]+\sum_{t^{\prime}=t+2}^{\tau} L_{i t^{\prime}}\left(\hat{r}_{i t^{\prime}}^{\lambda}\right) .
\end{aligned}
$$

Collecting the terms in the expression above shows that (16) holds.

We emphasize that although the optimality equation in (8) can be solved myopically for given Lagrange multipliers, the Lagrangian relaxation strategy is not entirely myopic because the Lagrange multipliers play the role of linking different time periods.

\subsection{Computing Lower Bounds on the Value Functions and Choosing a Good Set of Values for the Lagrange Multipliers}

We begin this section by showing that we obtain lower bounds on the value functions by solving the optimality equation in (4)-(6). A similar result is shown in Adelman and Mersereau [1] for infinite-horizon problems. Our proof is for finite-horizon problems, and it is considerably shorter.

PROPOSITION 3: If the Lagrange multipliers are positive, then we have $V_{t}^{L}\left(x_{t} \mid \lambda\right) \leq V_{t}\left(x_{t}\right)$ for all $t \in \mathcal{T}$.

PROOF: We show the result by induction over the time periods. It is easy to show the result for the last time period. Assuming that the result holds for time period $t+1$, we let $\hat{y}_{t}=\left\{\hat{y}_{i t}: i \in \mathcal{I} \cup\{\phi\}\right\}$ be the optimal solution to problem (1)-(3), in which case we have

$$
\begin{aligned}
V_{t}^{L}\left(x_{t} \mid \lambda\right) \leq & c_{\phi t}\left[\hat{y}_{\phi_{t}}-x_{\phi t}\right]+L_{\phi t}\left(\hat{y}_{\phi t}\right)+\sum_{i \in \mathcal{I}}\left[c_{i t}-\lambda_{i t}\right] \\
& \times\left[\hat{y}_{i t}-x_{i t}\right]+\sum_{i \in \mathcal{I}} L_{i t}\left(\hat{y}_{i t}\right)+\mathbb{E}\left\{V_{t+1}^{L}\left(\hat{y}_{t}-D_{t} \mid \lambda\right)\right\} \\
\leq & \sum_{i \in \mathcal{I} \cup\{\phi\}} c_{i t}\left[\hat{y}_{i t}-x_{i t}\right]+\sum_{i \in \mathcal{I} \cup\{\phi\}} L_{i t}\left(\hat{y}_{i t}\right) \\
& -\sum_{i \in \mathcal{I}} \lambda_{i t}\left[\hat{y}_{i t}-x_{i t}\right]+\mathbb{E}\left\{V_{t+1}\left(\hat{y}_{t}-D_{t}\right)\right\} \\
\leq & \sum_{i \in \mathcal{I} \cup\{\phi\}} c_{i t}\left[\hat{y}_{i t}-x_{i t}\right]+\sum_{i \in \mathcal{I} \cup\{\phi\}} L_{i t}\left(\hat{y}_{i t}\right) \\
& +\mathbb{E}\left\{V_{t+1}\left(\hat{y}_{t}-D_{t}\right)\right\},
\end{aligned}
$$

where the first inequality follows from the fact that the solution $\hat{y}_{t}=\left\{\hat{y}_{i t}: i \in \mathcal{I} \cup\{\phi\}\right\}$ satisfies constraints (5) and (6), the second inequality follows from the induction assumption, and the third inequality follows from the fact that $\lambda_{i t} \geq 0$ and $\hat{y}_{i t} \geq x_{i t}$ for all $i \in \mathcal{I}$. The result follows by noting that the last expression above is equal to $V_{t}\left(x_{t}\right)$.

Given that the initial state variable is $x_{1}$, the minimum expected cost over the whole planning horizon is $V_{1}\left(x_{1}\right)$. Proposition 3 implies that $V_{1}\left(x_{1}\right)$ is bounded from below by $V_{1}^{L}\left(x_{1} \mid \lambda\right)$ as long as the Lagrange multipliers are positive. Therefore, to obtain the tightest possible lower bound on $V_{1}\left(x_{1}\right)$, we can solve the problem

$$
\max _{\lambda \geq 0}\left\{V_{1}^{L}\left(x_{1} \mid \lambda\right)\right\}
$$

Proposition 4 below shows that $V_{1}^{L}\left(x_{1} \mid \cdot\right)$ has a subgradient so that by Theorem 3.2.6 in Bazaraa, Sherali and Shetty [4], $V_{1}^{L}\left(x_{1} \mid \lambda\right)$ is a concave function of the Lagrange multipliers. This implies that we can solve problem (17) by using 
standard subgradient optimization; see Section 6.3 in Bertsekas [5] and Section 10.3 in Wolsey [24]. The concavity of $V_{1}^{L}\left(x_{1} \mid \cdot\right)$ is shown in Adelman and Mersereau [1] for infinitehorizon problems, but Proposition 4 explicitly shows how to compute subgradients of $V_{1}^{L}\left(x_{1} \mid \cdot\right)$. We also emphasize that problem (17) provides a method for choosing a set of values for the Lagrange multipliers. Finally, we note that the optimal solution to problem (17) depends on the initial value of the state variable $x_{1}$, which is a fixed problem parameter.

We use some new notation to show that $V_{1}^{L}\left(x_{1} \mid \cdot\right)$ has a subgradient. Because $\left\{x_{i t}: i \in \mathcal{I}\right\}$ do not appear in the constraints in problem (4)-(6), the optimal solution to problem (4)-(6) does not depend on $\left\{x_{i t}: i \in \mathcal{I}\right\}$. As a function of the echelon inventory position at the warehouse and the Lagrange multipliers, we let $\hat{y}_{t}^{\lambda}\left(x_{\phi t}\right)=\left\{\hat{y}_{i t}^{\lambda}\left(x_{\phi t}\right): i \in \mathcal{I} \cup\{\phi\}\right\}$ be the optimal solution to problem (4)-(6). We consider a policy that makes the inventory replenishment decisions by solving problem (4)-(6) at every time period. In this case, the stochastic process $X_{\phi}^{\lambda}=\left\{X_{\phi t}^{\lambda}: t \in \mathcal{T}\right\}$ defined by $X_{\phi 1}^{\lambda}=x_{\phi 1}$ and

$$
X_{\phi, t+1}^{\lambda}=\hat{y}_{\phi t}^{\lambda}\left(X_{\phi t}^{\lambda}\right)-D_{\phi t}
$$

characterizes the echelon inventory position at the warehouse over the whole planning horizon. Similarly, the stochastic process $X_{i}^{\lambda}=\left\{X_{i t}^{\lambda}: t \in \mathcal{T}\right\}$ defined by $X_{i 1}^{\lambda}=x_{i 1}$ and

$$
X_{i, t+1}^{\lambda}=\hat{y}_{i t}^{\lambda}\left(X_{\phi t}^{\lambda}\right)-D_{i t}
$$

characterizes the echelon inventory position at retailer $i$ over the whole planning horizon. We note that the stochastic processes $\left\{X_{i}^{\lambda}: i \in \mathcal{I}\right\}$ depend on the stochastic process $X_{\phi}^{\lambda}$. We are now ready to prove that $V_{1}^{L}\left(x_{1} \mid \cdot\right)$ has a subgradient.

PROPOSITION 4: For two sets of Lagrange multipliers $\lambda$ and $\hat{\lambda}$, we have

$$
V_{1}^{L}\left(x_{1} \mid \hat{\lambda}\right) \leq V_{1}^{L}\left(x_{1} \mid \lambda\right)-\sum_{t \in \mathcal{T}} \sum_{i \in \mathcal{I}}\left[\hat{\lambda}_{i t}-\lambda_{i t}\right] \mathbb{E}\left\{\hat{y}_{i t}^{\lambda}\left(X_{\phi t}^{\lambda}\right)-X_{i t}^{\lambda}\right\} .
$$

PROOF: We use induction over the time periods to show that

$$
\begin{aligned}
V_{t}^{L}\left(x_{t} \mid \hat{\lambda}\right) \leq V_{t}^{L}\left(x_{t} \mid \lambda\right)- & \sum_{i \in \mathcal{I}}\left[\hat{\lambda}_{i t}-\lambda_{i t}\right]\left[\hat{y}_{i t}^{\lambda}\left(x_{\phi t}\right)-x_{i t}\right] \\
-\sum_{t^{\prime}=t+1}^{\tau} & \sum_{i \in \mathcal{I}}\left[\hat{\lambda}_{i t^{\prime}}-\lambda_{i t^{\prime}}\right] \\
& \quad \times \mathbb{E}\left\{\hat{y}_{i t^{\prime}}^{\lambda}\left(X_{\phi t^{\prime}}^{\lambda}\right)-X_{i t^{\prime}}^{\lambda} \mid X_{\phi t}^{\lambda}=x_{\phi t}\right\} .
\end{aligned}
$$

Using the expression above with $t=1$ completes the proof.

It is easy to show the result for the last time period. If we assume that the result holds for time period $t+1$, use it with $x_{t+1}=\hat{y}_{t}^{\lambda}\left(x_{\phi t}\right)-D_{t}$ and take the conditional expectations, then we have

$$
\begin{gathered}
\mathbb{E}\left\{V_{t+1}^{L}\left(\hat{y}_{t}^{\lambda}\left(x_{\phi t}\right)-D_{t} \mid \hat{\lambda}\right) \mid X_{\phi t}^{\lambda}=x_{\phi t}\right\} \\
\leq \mathbb{E}\left\{V_{t+1}^{L}\left(\hat{y}_{t}^{\lambda}\left(x_{\phi t}\right)-D_{t} \mid \lambda\right) \mid X_{\phi t}^{\lambda}=x_{\phi t}\right\} \\
-\sum_{i \in \mathcal{I}}\left[\hat{\lambda}_{i, t+1}-\lambda_{i, t+1}\right] \mathbb{E}\left\{\hat{y}_{i, t+1}^{\lambda}\left(\hat{y}_{\phi t}^{\lambda}\left(x_{\phi t}\right)-D_{\phi t}\right)\right. \\
\left.-\left[\hat{y}_{i t}^{\lambda}\left(x_{\phi t}\right)-D_{i t}\right] \mid X_{\phi t}^{\lambda}=x_{\phi t}\right\} \\
-\sum_{t^{\prime}=t+2}^{\tau} \sum_{i \in \mathcal{I}}\left[\hat{\lambda}_{i t^{\prime}}-\lambda_{i t^{\prime}}\right] \mathbb{E}\left\{\mathbb { E } \left\{\hat{y}_{i t^{\prime}}^{\lambda}\left(X_{\phi t^{\prime}}^{\lambda}\right)-X_{i t^{\prime}}^{\lambda} \mid X_{\phi, t+1}^{\lambda}\right.\right. \\
\left.\left.=\hat{y}_{\phi t}^{\lambda}\left(x_{\phi t}\right)-D_{\phi t}\right\} \mid X_{\phi t}^{\lambda}=x_{\phi t}\right\} .
\end{gathered}
$$

Because $X_{\phi t}^{\lambda}$ and $D_{t}$ are independent, the condition $X_{\phi t}^{\lambda}=$ $x_{\phi t}$ in the first two conditional expectations above can be dropped. Furthermore, since having $X_{\phi t}^{\lambda}=x_{\phi t}$ implies that $X_{\phi, t+1}^{\lambda}=\hat{y}_{\phi t}^{\lambda}\left(x_{\phi t}\right)-D_{\phi t}$, the last double expectation is equal to $\mathbb{E}\left\{\hat{y}_{i t^{\prime}}^{\lambda}\left(X_{\phi t^{\prime}}^{\lambda}\right)-X_{i t^{\prime}}^{\lambda} \mid X_{\phi t}^{\lambda}=x_{\phi t}\right\}$. Because $\hat{y}_{t}^{\lambda}\left(x_{\phi t}\right)$ is a feasible but not necessarily the optimal solution to problem (4)-(6) when we use the Lagrange multipliers $\hat{\lambda}$, we have

$$
\begin{gathered}
V_{t}^{L}\left(x_{t} \mid \hat{\lambda}\right) \leq c_{\phi t}\left[\hat{y}_{\phi_{t}}^{\lambda}\left(x_{\phi t}\right)-x_{\phi t}\right]+L_{\phi t}\left(\hat{y}_{\phi t}^{\lambda}\left(x_{\phi t}\right)\right) \\
+\sum_{i \in \mathcal{I}}\left[c_{i t}-\hat{\lambda}_{i t}\right]\left[\hat{y}_{i t}^{\lambda}\left(x_{\phi t}\right)-x_{i t}\right] \\
+\sum_{i \in \mathcal{I}} L_{i t}\left(\hat{y}_{i t}^{\lambda}\left(x_{\phi t}\right)\right)+\mathbb{E}\left\{V_{t+1}^{L}\left(\hat{y}_{t}^{\lambda}\left(x_{\phi t}\right)-D_{t} \mid \hat{\lambda}\right)\right\} \\
\leq c_{\phi t}\left[\hat{y}_{\phi_{t}}^{\lambda}\left(x_{\phi t}\right)-x_{\phi t}\right]+L_{\phi t}\left(\hat{y}_{\phi t}^{\lambda}\left(x_{\phi t}\right)\right) \\
+\sum_{i \in \mathcal{I}}\left[c_{i t}-\lambda_{i t}\right]\left[\hat{y}_{i t}^{\lambda}\left(x_{\phi t}\right)-x_{i t}\right]+\sum_{i \in \mathcal{I}} L_{i t}\left(\hat{y}_{i t}^{\lambda}\left(x_{\phi t}\right)\right) \\
-\sum_{i \in \mathcal{I}}\left[\hat{\lambda}_{i t}-\lambda_{i t}\right]\left[\hat{y}_{i t}^{\lambda}\left(x_{\phi t}\right)-x_{i t}\right]+\mathbb{E}\left\{V_{t+1}^{L}\left(\hat{y}_{t}^{\lambda}\left(x_{\phi t}\right)-D_{t} \mid \lambda\right)\right\} \\
-\sum_{i \in \mathcal{I}}\left[\hat{\lambda}_{i, t+1}-\lambda_{i, t+1}\right] \mathbb{E}\left\{\hat{y}_{i, t+1}^{\lambda}\left(\hat{y}_{\phi t}^{\lambda}\left(x_{\phi t}\right)-D_{\phi t}\right)\right. \\
\left.-\left[\hat{y}_{i t}^{\lambda}\left(x_{\phi t}\right)-D_{i t}\right] \mid X_{\phi t}^{\lambda}=x_{\phi t}\right\} \\
-\sum_{t^{\prime}=t+2}^{\tau} \sum_{i \in \mathcal{I}}\left[\hat{\lambda}_{i t^{\prime}}-\lambda_{i t^{\prime}}\right] \mathbb{E}\left\{\hat{y}_{i t^{\prime}}^{\lambda}\left(X_{\phi t^{\prime}}^{\lambda}\right)-X_{i t^{\prime}}^{\lambda} \mid X_{\phi t}^{\lambda}=x_{\phi t}\right\} \\
=V_{t}^{L}\left(x_{t} \mid \lambda\right)-\sum_{i \in \mathcal{I}}\left[\hat{\lambda}_{i t}-\lambda_{i t}\right]\left[\hat{y}_{i t}^{\lambda}\left(x_{\phi t}\right)-x_{i t}\right] \\
-\sum_{t^{\prime}=t+1}^{\tau} \sum_{i \in \mathcal{I}}\left[\hat{\lambda}_{i t^{\prime}}-\lambda_{i t^{\prime}}\right] \mathbb{E}\left\{\hat{y}_{i t^{\prime}}^{\lambda}\left(X_{\phi t^{\prime}}^{\lambda}\right)-X_{i t^{\prime}}^{\lambda} \mid X_{\phi t}^{\lambda}=x_{\phi t}\right\},
\end{gathered}
$$

where the second inequality follows from (21) and the equality follows from the fact that $\hat{y}_{t}^{\lambda}\left(x_{\phi t}\right)$ is the optimal solution to problem (4)-(6) and the definitions of $\left\{X_{i, t+1}^{\lambda}: i \in \mathcal{I} \cup\{\phi\}\right\}$ in (18) and (19).

In our computational experiments, we use discrete demand distributions with finite supports. In this case, the probability 
laws governing the stochastic processes $\left\{X_{i}^{\lambda}: i \in \mathcal{I} \cup\{\phi\}\right\}$ are characterized by finite-dimensional transition matrices and the expectation in (20) can easily be computed.

We close this section by emphasizing that the Lagrangian relaxation strategy is approximate because our Lagrange multipliers do not depend on the state variable. If we use a state-dependent Lagrange multiplier of the form $\lambda_{i t}\left(x_{t}\right)$, then it is possible to show that the Lagrangian relaxation strategy is exact and the optimal objective value of problem (17) is equal to $V_{1}\left(x_{1}\right)$. This observation suggests that we expect the error brought by the fact that our Lagrange multipliers do not depend on the state variable to be small whenever the trajectory of the state variable $\left\{x_{t}: t \in \mathcal{T}\right\}$ under the optimal inventory replenishment policy does not change too much from one sample path to another. From computational standpoint, we note that if we use a state-dependent Lagrange multiplier, then the number of Lagrange multipliers is as large as the number of possible states for the system and the Lagrangian relaxation strategy becomes intractable. Therefore, it is not practical to try to improve the lower bounds obtained by the Lagrangian relaxation strategy by switching from a state-independent to a state-dependent Lagrange multiplier.

\section{CLARK AND SCARF'S BALANCE ASSUMPTION}

In this section, we review the balance assumption of Clark and Scarf [8] and describe its practical significance. In their seminal work, Clark and Scarf [8] show that if their wellknown balance assumption is satisfied, then the value functions $\left\{V_{t}(\cdot): t \in \mathcal{T}\right\}$ computed through the optimality equation in (1)-(3) are separable functions of the form

$$
V_{t}^{B}\left(x_{t}\right)=\sum_{i \in \mathcal{I} \cup\{\phi\}} v_{i t}^{B}\left(x_{i t}\right)
$$

We shortly give a precise definition of the balance assumption. In the expression above, the value functions $\left\{v_{i t}^{B}(\cdot)\right.$ : $t \in \mathcal{T}\}$ for retailer $i$ are computed through the optimality equation

$$
\begin{aligned}
v_{i t}^{B}\left(x_{i t}\right)=\min _{y_{i t} \geq x_{i t}}\left\{c_{i t}\left[y_{i t}-x_{i t}\right]\right. & +L_{i t}\left(y_{i t}\right) \\
+ & \left.\mathbb{E}\left\{v_{i, t+1}^{B}\left(y_{i t}-D_{i t}\right)\right\}\right\} .
\end{aligned}
$$

This optimality equation computes the optimal inventory replenishment policy for retailer $i$ under the assumption that the warehouse has infinite supply. On the other hand, noting that the optimal solution to the problem

$$
\min _{y_{i t}}\left\{c_{i t}\left[y_{i t}-x_{i t}\right]+L_{i t}\left(y_{i t}\right)+\mathbb{E}\left\{v_{i, t+1}^{B}\left(y_{i t}-D_{i t}\right)\right\}\right\}
$$

does not depend on $x_{i t}$ and letting $\hat{r}_{i t}$ be the optimal solution to this problem, the value functions $\left\{v_{\phi t}^{B}(\cdot): t \in \mathcal{T}\right\}$ for the warehouse are computed through the optimality equation

$$
\begin{aligned}
v_{\phi t}^{B}\left(x_{\phi t}\right)=\min _{y_{\phi t} \geq x_{\phi t}}\left\{c_{\phi t}\left[y_{\phi t}-x_{\phi t}\right]+L_{\phi t}\left(y_{\phi t}\right)\right. \\
\left.+\mathbb{E}\left\{v_{\phi, t+1}^{B}\left(y_{\phi t}-D_{\phi t}\right)\right\}\right\}+\Delta_{t}^{B}\left(x_{\phi t}\right),
\end{aligned}
$$

where the functions $\left\{\Delta_{t}^{B}(\cdot): t \in \mathcal{T}\right\}$ are given by

$$
\begin{aligned}
\Delta_{t}^{B}\left(x_{\phi t}\right)=\min & \sum_{i \in \mathcal{I}} c_{i t}\left[y_{i t}-\hat{r}_{i t}\right] \\
& +\sum_{i \in \mathcal{I}} L_{i t}\left(y_{i t}\right)+\sum_{i \in \mathcal{I}} \mathbb{E}\left\{v_{i, t+1}^{B}\left(y_{i t}-D_{i t}\right)\right\} \\
& -\sum_{i \in \mathcal{I}} L_{i t}\left(\hat{r}_{i t}\right)-\sum_{i \in \mathcal{I}} \mathbb{E}\left\{v_{i, t+1}^{B}\left(\hat{r}_{i t}-D_{i t}\right)\right\} \\
\text { subject to } & \sum_{i \in \mathcal{I}} y_{i t} \leq x_{\phi t} .
\end{aligned}
$$

We encourage the reader to compare the optimality equation in (23) with the one in (8), the optimality equation in (25) with the one in (9) and the definition of $\Delta_{t}^{B}(\cdot)$ in problem (26)-(27) with the definition of $\Delta_{t}^{L}(\cdot \mid \lambda)$ in problem (10)-(11). The crucial difference between the Lagrangian relaxation strategy and the balance assumption is in the optimality equations in (8)-(23). The optimality equation in (8) implicitly tries to ensure that the shipment to retailer $i$ is nonnegative by deflating the per unit replenishment costs by $\left\{\lambda_{i t}: t \in \mathcal{T}\right\}$. On the other hand, the optimality equation in (23) ensures that the shipment to retailer $i$ is non-negative by imposing the constraint $y_{i t} \geq x_{i t}$.

We are now ready to give a precise definition of the balance assumption. The balance assumption amounts to the following two assumptions.

(A.1) When the echelon inventory position at the warehouse satisfies $x_{\phi t} \geq \sum_{i \in \mathcal{I}} \hat{r}_{i t}$, the constraint $\sum_{i \in \mathcal{I}} y_{i t} \leq x_{\phi t}$ in problem (1)-(3) is redundant.

(A.2) When the echelon inventory position at the warehouse satisfies $x_{\phi t}<\sum_{i \in \mathcal{I}} \hat{r}_{i t}$, the constraints $y_{i t} \geq x_{i t}$ for all $i \in \mathcal{I}$ in problem (1)-(3) are redundant.

Under the balance assumption, (A.2) implies that we do not have to impose the constraints $y_{i t} \geq x_{i t}$ for all $i \in \mathcal{I}$ in problem (1)-(3) when we have $x_{\phi t}<\sum_{i \in \mathcal{I}} \hat{r}_{i t}$. We note that the constraints $y_{i t} \geq x_{i t}$ for all $i \in \mathcal{I}$ ensure the nonnegativity of the shipments to the retailers and sending a negative shipment from the warehouse to a retailer is equivalent to withdrawing the inventory from the retailer back to the warehouse, at which point the inventory withdrawn back to the warehouse can be distributed to another retailer. Therefore, (A.2) essentially states that when we have $x_{\phi t}<\sum_{i \in \mathcal{I}} \hat{r}_{i t}$, it is not desirable to redistribute the total amount of retailer inventory among the retailers even if it is allowed to do so. 
The next proposition shows that the value functions computed through the optimality equation in (1)-(3) are separable functions of the form (22) as long as the balance assumption holds. This result is briefly sketched in Clark and Scarf [8], but we provide a rigorous proof here.

PROPOSITION 5: Assume that the value functions $\left\{V_{t}(\cdot): t \in \mathcal{T}\right\},\left\{v_{i t}^{B}(\cdot): i \in \mathcal{I}, t \in \mathcal{T}\right\}$, and $\left\{v_{\phi t}^{B}(\cdot): t \in \mathcal{T}\right\}$ are respectively computed through the optimality equations in (1)-(3), (23), and (25). If (A.1)-(A.2) hold and $\left\{V_{t}^{B}(\cdot)\right.$ : $t \in \mathcal{T}\}$ are as defined in (22), then we have $V_{t}\left(x_{t}\right)=V_{t}^{B}\left(x_{t}\right)$ for all $t \in \mathcal{T}$.

PROOF: We show the result by induction over the time periods. It is easy to show the result for the last time period. Assuming that the result holds for time period $t+1$, we consider two cases.

CASE 1: Assume that $x_{\phi t} \geq \sum_{i \in \mathcal{I}} \hat{r}_{i t}$. Using the induction assumption and noting that the constraint $\sum_{i \in \mathcal{I}} y_{i t} \leq x_{\phi t}$ in problem (1)-(3) is redundant, we have

$$
\begin{aligned}
V_{t}\left(x_{t}\right)=\min & \sum_{i \in \mathcal{I} \cup\{\phi\}} c_{i t}\left[y_{i t}-x_{i t}\right]+\sum_{i \in \mathcal{I} \cup\{\phi\}} L_{i t}\left(y_{i t}\right) \\
& +\sum_{i \in \mathcal{I} \cup\{\phi\}} \mathbb{E}\left\{v_{i, t+1}^{B}\left(y_{i t}-D_{i t}\right)\right\}
\end{aligned}
$$

subject to $\quad y_{i t} \geq x_{i t} \quad$ for all $i \in \mathcal{I} \cup\{\phi\}$.

The problem above decomposes by the installations, and noting (23) and (25), we have

$$
V_{t}\left(x_{t}\right)=v_{\phi t}\left(x_{\phi t}\right)-\Delta_{t}^{B}\left(x_{\phi t}\right)+\sum_{i \in \mathcal{I}} v_{i t}^{B}\left(x_{i t}\right)
$$

We conclude this part by showing that $\Delta_{t}^{B}\left(x_{\phi t}\right)=0$ when we have $x_{\phi t} \geq \sum_{i \in \mathcal{I}} \hat{r}_{i t}$. Because $\hat{r}_{i t}$ is the optimal solution to problem (24), the unconstrained minimizer of the objective function of problem (26)-(27) is $\left\{\hat{r}_{i t}: i \in \mathcal{I}\right\}$. The solution $\left\{\hat{r}_{i t}: i \in \mathcal{I}\right\}$ is also feasible to problem (26)-(27). Therefore, the optimal solution to problem (26)-(27) is $\left\{\hat{r}_{i t}: i \in \mathcal{I}\right\}$ and we have $\Delta_{t}^{B}\left(x_{\phi t}\right)=0$.

CASE 2: Assume that $x_{\phi t}<\sum_{i \in \mathcal{I}} \hat{r}_{i t}$. Using the induction assumption and noting that the constraints $y_{i t} \geq x_{i t}$ for all $i \in \mathcal{I}$ in problem (1)-(3) are redundant, we have

$$
\begin{aligned}
V_{t}\left(x_{t}\right)=\min & \sum_{i \in \mathcal{I} \cup\{\phi\}} c_{i t}\left[y_{i t}-x_{i t}\right]+\sum_{i \in \mathcal{I} \cup\{\phi\}} L_{i t}\left(y_{i t}\right) \\
& +\sum_{i \in \mathcal{I} \cup\{\phi\}} \mathbb{E}\left\{v_{i, t+1}^{B}\left(y_{i t}-D_{i t}\right)\right\} \\
\text { subject to } & \sum_{i \in \mathcal{I}} y_{i t} \leq x_{\phi t} \\
& y_{\phi t} \geq x_{\phi t} .
\end{aligned}
$$

If we write the objective function of problem (28)-(30) as

$$
\begin{gathered}
c_{\phi t}\left[y_{\phi t}-x_{\phi t}\right]+L_{\phi t}\left(y_{\phi t}\right)+\mathbb{E}\left\{v_{\phi, t+1}^{B}\left(y_{\phi t}-D_{\phi t}\right)\right\} \\
+\sum_{i \in \mathcal{I}} c_{i t}\left[y_{i t}-\hat{r}_{i t}\right] \\
+\sum_{i \in \mathcal{I}} L_{i t}\left(y_{i t}\right)+\sum_{i \in \mathcal{I}} \mathbb{E}\left\{v_{i, t+1}^{B}\left(y_{i t}-D_{i t}\right)\right\} \\
-\sum_{i \in \mathcal{I}} L_{i t}\left(\hat{r}_{i t}\right)-\sum_{i \in \mathcal{I}} \mathbb{E}\left\{v_{i, t+1}^{B}\left(\hat{r}_{i t}-D_{i t}\right)\right\} \\
+\sum_{i \in \mathcal{I}} c_{i t}\left[\hat{r}_{i t}-x_{i t}\right]+\sum_{i \in \mathcal{I}} L_{i t}\left(\hat{r}_{i t}\right) \\
+\sum_{i \in \mathcal{I}} \mathbb{E}\left\{v_{i, t+1}^{B}\left(\hat{r}_{i t}-D_{i t}\right)\right\},
\end{gathered}
$$

then problems (26)-(27) and (28)-(30) imply that

$$
\begin{array}{r}
V_{t}\left(x_{t}\right)=\min _{y_{\phi t} \geq x_{\phi t}}\left\{c_{\phi t}\left[y_{\phi t}-x_{\phi t}\right]+L_{\phi t}\left(y_{\phi t}\right)\right. \\
\left.+\mathbb{E}\left\{v_{\phi, t+1}^{B}\left(y_{\phi t}-D_{\phi t}\right)\right\}\right\}+\Delta_{t}^{B}\left(x_{\phi t}\right) \\
+\sum_{i \in \mathcal{I}} c_{i t}\left[\hat{r}_{i t}-x_{i t}\right]+\sum_{i \in \mathcal{I}} L_{i t}\left(\hat{r}_{i t}\right)+\sum_{i \in \mathcal{I}} \mathbb{E}\left\{v_{i, t+1}^{B}\left(\hat{r}_{i t}-D_{i t}\right)\right\} \\
=v_{\phi t}^{B}\left(x_{\phi t}\right)+\sum_{i \in \mathcal{I}} c_{i t}\left[\hat{r}_{i t}-x_{i t}\right]+\sum_{i \in \mathcal{I}} L_{i t}\left(\hat{r}_{i t}\right) \\
+\sum_{i \in \mathcal{I}} \mathbb{E}\left\{v_{i, t+1}^{B}\left(\hat{r}_{i t}-D_{i t}\right)\right\} .
\end{array}
$$

By Lemma 7 in Appendix 9, if $x_{\phi t}<\sum_{i \in \mathcal{I}} \hat{r}_{i t}$ and (A2) holds, then we have $v_{i t}^{B}\left(x_{i t}\right)=c_{i t}\left[\hat{r}_{i t}-x_{i t}\right]+L_{i t}\left(\hat{r}_{i t}\right)+$ $\mathbb{E}\left\{v_{i, t+1}^{B}\left(\hat{r}_{i t}-D_{i t}\right)\right\}$ for all $i \in \mathcal{I}$ and the result follows.

Proposition 5 shows that under the balance assumption, we can compute the value functions by focusing on one installation at a time. In particular, we first solve the optimality equation in (23) to compute $\left\{v_{i t}^{B}(\cdot): i \in \mathcal{I}, t \in \mathcal{T}\right\}$. In this case, we can compute $\left\{\Delta_{t}^{B}(\cdot): t \in \mathcal{T}\right\}$ by solving problem (26)-(27). Finally, we can compute $\left\{v_{\phi t}^{B}(\cdot): t \in \mathcal{T}\right\}$ by solving the optimality equation in (25). As long as the balance assumption holds, $\left\{V_{t}^{B}(\cdot): t \in \mathcal{T}\right\}$ coincides with $\left\{V_{t}(\cdot): t \in \mathcal{T}\right\}$.

Clearly, it is not realistic to expect that the balance assumption always holds. Furthermore, it is not easy to check the balance assumption for a particular problem instance. Nevertheless, even if the balance assumption does not hold, we can still solve the optimality equations in (23) and (25) to compute $\left\{V_{t}^{B}(\cdot): t \in \mathcal{T}\right\}$. The next proposition shows that irrespective of whether the balance assumption holds or not, $\left\{V_{t}^{B}(\cdot): t \in \mathcal{T}\right\}$ always provide lower bounds on $\left\{V_{t}(\cdot): t \in \mathcal{T}\right\}$. Clark and Scarf [8] mention this result without a proof, and we provide a rigorous proof here. 
PROPOSITION 6: We have $V_{t}^{B}\left(x_{t}\right) \leq V_{t}\left(x_{t}\right)$ for all $t \in \mathcal{T}$.

PROOF: We show the result by induction over the time periods. It is easy to show the result for the last time period. Assuming that the result holds for time period $t+1$, and using the optimality equation in (1)-(3) and the induction assumption, we have

$$
\begin{aligned}
V_{t}\left(x_{t}\right) \geq \min & \sum_{i \in \mathcal{I} \cup\{\phi\}} c_{i t}\left[y_{i t}-x_{i t}\right]+\sum_{i \in \mathcal{I} \cup\{\phi\}} L_{i t}\left(y_{i t}\right) \\
& +\sum_{i \in \mathcal{I} \cup\{\phi\}} \mathbb{E}\left\{v_{i, t+1}^{B}\left(y_{i t}-D_{i t}\right)\right\}
\end{aligned}
$$

subject to (2), (3).

The problem on the right side above decomposes into two problems. The first problem has the form

$$
\min _{y_{\phi t} \geq x_{\phi t}}\left\{c_{\phi t}\left[y_{\phi t}-x_{\phi t}\right]+L_{\phi t}\left(y_{\phi t}\right)+\mathbb{E}\left\{v_{\phi, t+1}^{B}\left(y_{\phi t}-D_{\phi t}\right)\right\}\right\}
$$

and the second problem has the same form

$$
\begin{aligned}
\min & \sum_{i \in \mathcal{I}} c_{i t}\left[y_{i t}-x_{i t}\right]+\sum_{i \in \mathcal{I}} L_{i t}\left(y_{i t}\right) \\
& +\sum_{i \in \mathcal{I}} \mathbb{E}\left\{v_{i, t+1}^{B}\left(y_{i t}-D_{i t}\right)\right\} \\
\text { subject to } & \sum_{i \in \mathcal{I}} y_{i t} \leq x_{\phi t} \\
& y_{i t} \geq x_{i t} \quad \text { for all } i \in \mathcal{I} .
\end{aligned}
$$

Lemma 8 in Appendix 9 shows that

$$
\begin{aligned}
\Delta_{t}^{B}\left(x_{\phi t}\right)+\sum_{i \in \mathcal{I}} \min _{y_{i t} \geq x_{i t}}\left\{c_{i t}\left[y_{i t}-x_{i t}\right]\right. & +L_{i t}\left(y_{i t}\right) \\
& \left.+\mathbb{E}\left\{v_{i, t+1}^{B}\left(y_{i t}-D_{i t}\right)\right\}\right\}
\end{aligned}
$$

provides a lower bound on the optimal objective value of problem (31)-(33). Therefore, we obtain

$$
\begin{array}{r}
V_{t}\left(x_{t}\right) \geq \min _{y_{\phi t} \geq x_{\phi t}}\left\{c_{\phi t}\left[y_{\phi t}-x_{\phi t}\right]+L_{\phi t}\left(y_{\phi t}\right)\right. \\
\left.+\mathbb{E}\left\{v_{\phi, t+1}^{B}\left(y_{\phi t}-D_{\phi t}\right)\right\}\right\} \\
+\Delta_{t}^{B}\left(x_{\phi t}\right)+\sum_{i \in \mathcal{I}} \min _{y_{i t} \geq x_{i t}}\left\{c_{i t}\left[y_{i t}-x_{i t}\right]+L_{i t}\left(y_{i t}\right)\right. \\
\left.+\mathbb{E}\left\{v_{i, t+1}^{B}\left(y_{i t}-D_{i t}\right)\right\}\right\} .
\end{array}
$$

By (23) and (25), the expression on the right side above is equal to $\sum_{i \in \mathcal{I} \cup\{\phi\}} v_{i t}^{B}\left(x_{i t}\right)$ and the result follows.

\section{FEDERGRUEN AND ZIPKIN'S RELAXATION STRATEGY}

Similar to the Lagrangian relaxation strategy that we propose in this work, the relaxation strategy of Federgruen and Zipkin [14] relaxes the constraints $y_{i t} \geq x_{i t}$ for all $i \in \mathcal{I}$ in problem (1)-(3), but it does not associate Lagrange multipliers with them. Therefore, the relaxation strategy of Federgruen and Zipkin [14] can be obtained by following the material in Section 3 under the assumption that all of the Lagrange multipliers are equal to zero. In other words, if we use the notation in Section 3, then the relaxation strategy of Federgruen and Zipkin [14] uses $\left\{V_{t}^{L}(\cdot \mid 0): t \in \mathcal{T}\right\}$ as lower bounds on $\left\{V_{t}(\cdot): t \in \mathcal{T}\right\}$. Because Proposition 3 continues to hold when all of the Lagrange multipliers are equal to zero, the relaxation strategy of Federgruen and Zipkin [14] indeed comes up with lower bounds on the value functions.

\section{COMPARISON OF THE LOWER BOUNDS ON THE VALUE FUNCTIONS}

In this section, we compare the lower bounds on the value functions computed under our Lagrangian relaxation strategy, the balance assumption of Clark and Scarf [8] and the relaxation strategy of Federgruen and Zipkin [14].

Because $\lambda=0$ is a feasible solution to problem (17), the lower bounds computed under the Lagrangian relaxation strategy are at least as tight as those computed under the relaxation strategy of Federgruen and Zipkin [14]. Our computational experiments indicate that there can be quite substantial gaps between the lower bounds computed under the Lagrangian relaxation strategy and the relaxation strategy of Federgruen and Zipkin [14].

It turns out that there does not exist a consistent ordering between the lower bounds computed under the Lagrangian relaxation strategy and the balance assumption. Under the balance assumption, we assume that the constraints $y_{i t} \geq x_{i t}$ for all $i \in \mathcal{I}$ are redundant only when $x_{\phi t}<\sum_{i \in \mathcal{I}} \hat{r}_{i t}$ holds, but no penalty is associated with assuming that these constraints are redundant. On the other hand, the Lagrangian relaxation strategy always relaxes the constraints $y_{i t} \geq x_{i t}$ for all $i \in \mathcal{I}$, but it associates Lagrange multipliers with them. In Appendix B, we give two examples that clearly show that neither of the lower bounds computed under the Lagrangian relaxation strategy and the balance assumption is consistently superior. It is, in fact, not too difficult to generate such examples. In particular, if the demands at the retailers are deterministic, then the Lagrangian relaxation strategy reduces to standard Lagrangian relaxation for deterministic optimization problems and the lower bounds computed under the Lagrangian relaxation strategy become tight. Also, by choosing the initial echelon inventory position $x_{\phi 1}$ at the warehouse carefully, it is possible to generate problem 
instances with deterministic demands at the retailers where the balance assumption fails. Therefore, one can generate problem instances where the lower bounds computed under the Lagrangian relaxation strategy are strictly tighter than the lower bounds computed under the balance assumption. On the other hand, Clark and Scarf [8] show that for problem instances with one retailer, the lower bounds computed under the balance assumption are tight, but there is no such guarantee for the Lagrangian relaxation strategy. Therefore, one can generate problem instances with one retailer where the lower bounds computed under the balance assumption are strictly tighter than the lower bounds computed under the Lagrangian relaxation strategy.

The remark that immediately precedes Proposition 5 suggests that the balance assumption may not be satisfied when it is desirable to redistribute the total amount of retailer inventory among the retailers if it were allowed to do so. One may conjecture numerous situations where it would indeed be desirable to redistribute the total amount of retailer inventory among the retailers. For example, if the holding cost at the warehouse or the backlogging cost at the retailers is high, then it is reasonable to expect that a major portion of the inventory would be held at the retailers rather than at the warehouse. In this case, a high demand at one retailer and a low demand at another retailer at a particular time period may leave the first retailer in short supply, and it may be desirable to redistribute the excess inventory at the second retailer to the first retailer if it were allowed to do so. It may also be desirable to redistribute the total amount of retailer inventory among the retailers when the demands at the retailers are highly nonstationary. In particular, if the expected demand at one retailer is unusually high at one time period, then it is conceivable that this retailer receives a large replenishment quantity at the current time period. If, by chance, the demand at this retailer at the current time period turns out to be low and the expected demand at another retailer at the next time period is unusually high, then it may be desirable to redistribute the excess inventory at the first retailer to the second retailer. It is, in fact, such imbalance of inventories among the retailers that gives the balance assumption its name. That is, the balance assumption amounts to assuming that such imbalance of inventories among the retailers does not occur. The computational experiments in Section 8 confirm our expectations and indicate that the relative performance of the balance assumption becomes less satisfactory when the holding cost at the warehouse or the backlogging cost at the retailers is high and when the demands at the retailers are highly nonstationary.

We also note that using the Lagrangian relaxation strategy by setting all the Lagrange multipliers to zero is not equivalent to computing the lower bounds under the balance assumption. In other words, we do not necessarily have $V_{1}^{L}\left(x_{1} \mid 0\right)=V_{1}^{B}\left(x_{1}\right)$. This can easily be seen by noting that the constraint $y_{i t} \geq x_{i t}$ appears in problem (23), but not in problem (8). Therefore, the relaxation ideas used by Federgruen and Zipkin [14], which are equivalent to setting all the Lagrange multipliers to zero, are different from the balance assumption of Clark and Scarf [8]. We emphasize this distinction because the ideas used by Clark and Scarf [8] and Federgruen and Zipkin [14] are sometimes both referred to as the balance assumption; see, for example, Jackson [18]. Although these ideas share similarities, they are not equivalent to each other. In Appendix B, we give an example where we have $V_{1}^{L}\left(x_{1} \mid 0\right)<V_{1}^{B}\left(x_{1}\right)$.

\section{APPLYING THE INVENTORY REPLENISHMENT POLICIES}

In this section, we examine the structure of the inventory replenishment policies obtained under the Lagrangian relaxation strategy and the balance assumption. Letting $\hat{\lambda}$ be the optimal solution to problem (17), the value functions $\left\{V_{t}^{L}(\cdot \mid \hat{\lambda}): t \in \mathcal{T}\right\}$ computed under the Lagrangian relaxation strategy and the value functions $\left\{V_{t}^{B}(\cdot): t \in \mathcal{T}\right\}$ computed under the balance assumption are separable functions of the form $\left\{\sum_{i \in \mathcal{I} \cup\{\phi\}} \vartheta_{i t}(\cdot): t \in \mathcal{T}\right\}$; see (7) and (22). Furthermore, it can be shown that the functions $\left\{\vartheta_{i t}(\cdot): i \in\right.$ $\mathcal{I} \cup\{\phi\}, t \in \mathcal{T}\}$ are convex.

We make the inventory replenishment decisions by replacing the value functions $\left\{V_{t}(\cdot): t \in \mathcal{T}\right\}$ in problem (1)-(3) with $\left\{\sum_{i \in \mathcal{I} \cup\{\phi\}} \vartheta_{i t}(\cdot): t \in \mathcal{T}\right\}$. In this case, problem (1)-(3) decompose into two problems, one for the retailers and one for the warehouse. The problem for the retailers has the form

$$
\begin{aligned}
\min & \sum_{i \in \mathcal{I}} c_{i t}\left[y_{i t}-x_{i t}\right]+\sum_{i \in \mathcal{I}} L_{i t}\left(y_{i t}\right) \\
& +\sum_{i \in \mathcal{I}} \mathbb{E}\left\{\vartheta_{i, t+1}\left(y_{i t}-D_{i t}\right)\right\} \\
\text { subject to } & \sum_{i \in \mathcal{I}} y_{i t} \leq x_{\phi t} \\
& y_{i t} \geq x_{i t} \quad \text { for all } i \in \mathcal{I},
\end{aligned}
$$

whereas the problem for the warehouse has the form

$$
\begin{aligned}
\min _{y_{\phi t} \geq x_{\phi t}}\left\{c_{\phi t}\left[y_{\phi t}-x_{\phi t}\right]+L_{\phi t}\left(y_{\phi t}\right)\right. & \\
& \left.+\mathbb{E}\left\{\vartheta_{\phi, t+1}\left(y_{\phi t}-D_{\phi t}\right)\right\}\right\} .
\end{aligned}
$$

In our computational experiments, we use test problems that involve discrete demand distributions. In this case, one can show that the functions $\left\{\vartheta_{i t}(\cdot): i \in \mathcal{I} \cup\{\phi\}, t \in \mathcal{T}\right\}$ are piecewise-linear convex, and we can easily solve the two problems above by using simple marginal analysis; see Fox [16]. For example, in problem (34)-(36), we can start with the feasible solution $\tilde{y}_{t}=\left\{\tilde{y}_{i t}: i \in \mathcal{I}\right\}$ with $\tilde{y}_{i t}=x_{i t}$ for all $i \in \mathcal{I}$ and iteratively increase one component of this solution that yields the largest decrease in the objective function 

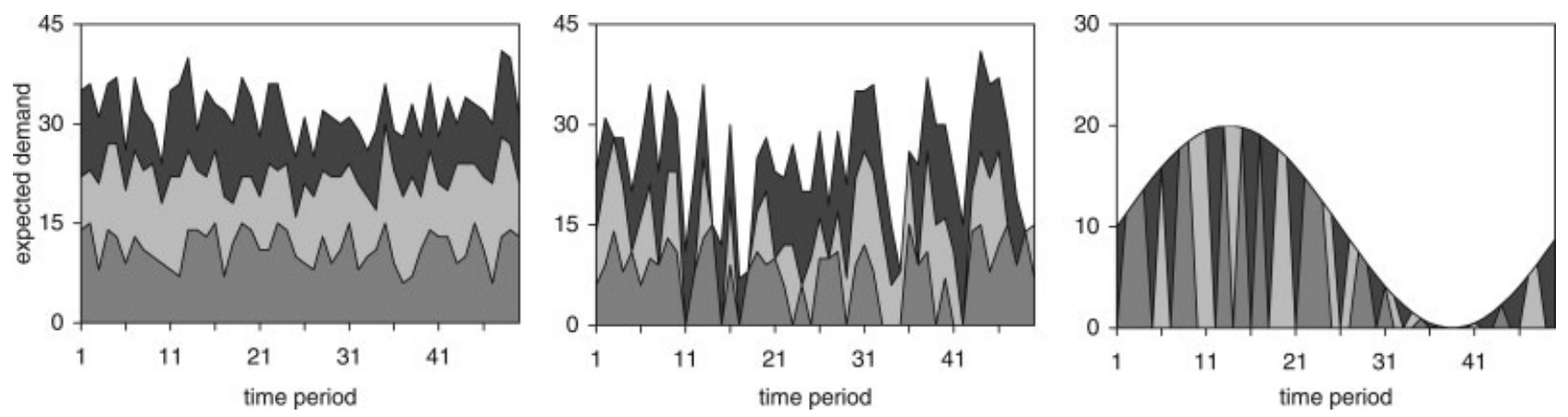

Figure 1. Expected demands at different installations over the planning horizon for particular instances of demand profiles $\mathcal{D}_{1}, \mathcal{D}_{2}$, and $\mathcal{D}_{3}$.

value. We repeat this until constraint (35) becomes tight or increasing one component of the solution does not yield a decrease in the objective function value.

\section{COMPUTATIONAL EXPERIMENTS}

In this section, we numerically compare the performance of the inventory replenishment policies obtained under the Lagrangian relaxation strategy with those obtained under the balance assumption of Clark and Scarf [8] and the relaxation strategy of Federgruen and Zipkin [14].

\subsection{Experimental Setup and Benchmarks}

In our experimental setup, we consider 21 test problems in a base case and systematically vary the attributes of these test problems to investigate the effects of different problem parameters. In the base case, we consider a distribution system with three retailers. The planning horizon includes 50 time periods. The holding and backlogging costs are $h_{\phi t}=0.6$, $h_{i t}=1$, and $b_{i t}=19$ for all $i \in \mathcal{I}$ and $t \in \mathcal{T}$. The replenishment order shipped to a certain installation at a certain time period reaches the installation at the next time period. We note that this is in contrast with our earlier assumption that the lead times for all replenishments are zero. We assume that the demand at retailer $i$ at time period $t$ has a Poisson distribution with mean $\alpha_{i t}$. We generate three demand profiles by generating $\left\{\alpha_{i t}: i \in \mathcal{I}, t \in \mathcal{T}\right\}$ in three different ways. For demand profile $\mathcal{D}_{1}$, we generate $\alpha_{i t}$ from the uniform distribution over [5, 15]. For demand profile $\mathcal{D}_{2}$, we set $\alpha_{i t}=0$ with probability $1 / 4$, but otherwise, generate $\alpha_{i t}$ from the uniform distribution over $[5,15]$. For demand profile $\mathcal{D}_{3}$, we generate $i_{t}$ from the uniform distribution over $\mathcal{I}$ and set $\alpha_{i t}=10 \mathbf{1}\left(i_{t}=i\right)(1+\sin (2 \pi t / \tau))$, where $\mathbf{1}(\cdot)$ is the indicator function. Demand profile $\mathcal{D}_{1}$ characterizes a situation where the demand distributions at the retailers and the warehouse are relatively stationary. Demand profile $\mathcal{D}_{2}$ allows time periods where a retailer does not face any demand, and it introduces additional nonstationarity when compared with demand profile $\mathcal{D}_{1}$. Such nonstationarity may occur when the retailers do not face demand at known time periods. The expected demand at the warehouse under demand profile $\mathcal{D}_{3}$ is $10(1+\sin (2 \pi t / \tau))$, and it has a seasonal component that changes smoothly, but each retailer faces demand at time periods that are different from the others. The role of demand profile $\mathcal{D}_{3}$ is to test the different solution methods under highly nonstationary demands. In particular, this demand profile involves nonstationarity in both the expected demand at the warehouse and the expected demand at the retailers. Furthermore, because this demand profile has exactly one retailer facing demand at each time period, it becomes more crucial to make the "correct" inventory allocation decisions. We obtain seven instances of demand profiles $\mathcal{D}_{1}, \mathcal{D}_{2}$, and $\mathcal{D}_{3}$ by using seven different random seeds to generate $\left\{\alpha_{i t}: i \in \mathcal{I}, t \in \mathcal{T}\right\}$ and this gives the 21 test problems in the base case. We note that once we generate $\left\{\alpha_{i t}: i \in \mathcal{I}, t \in \mathcal{T}\right\}$, these values are known to the decision maker. The area charts in Fig. 1 plot $\left\{\alpha_{i t}: i \in \mathcal{I}, t \in \mathcal{T}\right\}$ for particular instances of demand profiles $\mathcal{D}_{1}, \mathcal{D}_{2}$, and $\mathcal{D}_{3}$. The horizontal axis in each chart corresponds to the time periods in the planning horizon and the areas correspond to the retailers. We stack the areas so that the outline of the top area shows the expected demand at the warehouse.

For each instance of the demand profiles, we vary one problem parameter at a time to investigate how that problem parameter affects the performance of the benchmarks. In particular, we vary the holding cost at the warehouse, the backlogging cost at the retailers, the number of retailers, the length of the planning horizon, and the length of the lead time.

For the Lagrangian relaxation strategy, we use subgradient optimization to solve problem (17). We take the step size at iteration $k$ as $20 / \sqrt{k}$ and terminate the subgradient search after 1000 iterations. These settings provide stable performance and good solutions, although the step size that we use does not guarantee convergence. After obtaining the optimal solution $\hat{\lambda}$ to problem (17), we let $\vartheta_{i t}(\cdot)=v_{i t}^{L}(\cdot \mid \hat{\lambda})$ for 
Table 1. Detailed performance of LR, BA and RS for the 21 test problems in the base case.

\begin{tabular}{|c|c|c|c|c|c|c|c|c|c|c|}
\hline \multirow[b]{2}{*}{ Prob. } & \multicolumn{3}{|c|}{ Lower bnd. } & \multicolumn{2}{|c|}{ LR vs. } & \multicolumn{3}{|c|}{ Exp. cost } & \multicolumn{2}{|c|}{ LR vs. } \\
\hline & LR & BA & RS & BA & $\mathrm{RS}$ & LR & BA & $\mathrm{RS}$ & BA & $\mathrm{RS}$ \\
\hline$\left(\mathcal{D}_{1}, 1\right)$ & 2651 & 2651 & 2651 & 0.00 & 0.00 & 2660 & 2651 & 2660 & -0.34 & 0.00 \\
\hline$\left(\mathcal{D}_{1}, 2\right)$ & 2551 & 2560 & 2551 & -0.35 & 0.00 & 2562 & 2562 & 2562 & 0.01 & 0.00 \\
\hline$\left(\mathcal{D}_{1}, 3\right)$ & 2680 & 2680 & 2680 & 0.00 & 0.00 & 2680 & 2681 & 2680 & 0.02 & 0.00 \\
\hline$\left(\mathcal{D}_{1}, 4\right)$ & 2651 & 2651 & 2651 & 0.00 & 0.00 & 2656 & 2656 & 2656 & -0.01 & 0.00 \\
\hline$\left(\mathcal{D}_{1}, 5\right)$ & 2714 & 2713 & 2714 & 0.03 & 0.00 & 2714 & 2714 & 2714 & 0.00 & 0.00 \\
\hline$\left(\mathcal{D}_{1}, 6\right)$ & 2675 & 2675 & 2675 & 0.00 & 0.00 & 2675 & 2676 & 2675 & 0.01 & 0.00 \\
\hline$\left(\mathcal{D}_{1}, 7\right)$ & 2614 & 2613 & 2614 & 0.03 & 0.00 & 2615 & 2615 & 2615 & 0.00 & 0.00 \\
\hline avg. & & & & -0.04 & 0.00 & & & & -0.04 & 0.00 \\
\hline$\left(\mathcal{D}_{2}, 1\right)$ & 1906 & 1892 & 1822 & 0.72 & 4.57 & 2006 & 2091 & 2160 & 4.05 & 7.12 \\
\hline$\left(\mathcal{D}_{2}, 2\right)$ & 1947 & 1934 & 1886 & 0.63 & 3.23 & 2019 & 2099 & 2152 & 3.81 & 6.18 \\
\hline$\left(\mathcal{D}_{2}, 3\right)$ & 1809 & 1797 & 1736 & 0.68 & 4.21 & 1870 & 1961 & 2006 & 4.61 & 6.76 \\
\hline$\left(\mathcal{D}_{2}, 4\right)$ & 2111 & 2094 & 2022 & 0.80 & 4.42 & 2228 & 2363 & 2493 & 5.71 & 10.61 \\
\hline$\left(\mathcal{D}_{2}, 5\right)$ & 2235 & 2223 & 2153 & 0.54 & 3.76 & 2314 & 2404 & 2463 & 3.75 & 6.06 \\
\hline$\left(\mathcal{D}_{2}, 6\right)$ & 1746 & 1729 & 1648 & 0.99 & 5.99 & 1871 & 2005 & 2133 & 6.71 & 12.30 \\
\hline$\left(\mathcal{D}_{2}, 7\right)$ & 1602 & 1593 & 1533 & 0.54 & 4.48 & 1692 & 1752 & 1816 & 3.44 & 6.81 \\
\hline avg. & & & & 0.70 & 4.38 & & & & 4.58 & 7.98 \\
\hline$\left(\mathcal{D}_{3}, 1\right)$ & 923 & 896 & 765 & 3.06 & 20.66 & 1420 & 1572 & 1803 & 9.69 & 21.26 \\
\hline$\left(\mathcal{D}_{3}, 2\right)$ & 922 & 895 & 765 & 2.95 & 20.48 & 1415 & 1563 & 1788 & 9.48 & 20.84 \\
\hline$\left(\mathcal{D}_{3}, 3\right)$ & 928 & 896 & 765 & 3.63 & 21.33 & 1466 & 1618 & 1818 & 9.38 & 19.38 \\
\hline$\left(\mathcal{D}_{3}, 4\right)$ & 918 & 899 & 765 & 2.12 & 19.95 & 1378 & 1511 & 1732 & 8.83 & 20.44 \\
\hline$\left(\mathcal{D}_{3}, 5\right)$ & 932 & 895 & 765 & 4.11 & 21.85 & 1433 & 1566 & 1754 & 8.48 & 18.27 \\
\hline$\left(\mathcal{D}_{3}, 6\right)$ & 930 & 901 & 765 & 3.17 & 21.52 & 1424 & 1649 & 1903 & 13.61 & 25.15 \\
\hline$\left(\mathcal{D}_{3}, 7\right)$ & 935 & 905 & 765 & 3.27 & 22.17 & 1497 & 1620 & 1850 & 7.61 & 19.10 \\
\hline Avg. & & & & 3.19 & 21.13 & & & & 9.58 & 20.64 \\
\hline
\end{tabular}

all $i \in \mathcal{I} \cup\{\phi\}$ and $t \in \mathcal{T}$ and solve problems (34)-(36) and (37) to make the inventory replenishment decisions. We refer to this solution method as LR, standing for Lagrangian relaxation.

We use the balance assumption of Clark and Scarf [8] and the relaxation strategy of Federgruen and Zipkin [14] as benchmarks. Under the balance assumption, we solve the optimality equations in (23) and (25) to compute $\left\{v_{i t}^{B}(\cdot)\right.$ : $i \in \mathcal{I} \cup\{\phi\}, t \in \mathcal{T}\}$. After this, we let $\vartheta_{i t}(\cdot)=v_{i t}^{B}(\cdot)$ for all $i \in \mathcal{I} \cup\{\phi\}$ and $t \in \mathcal{T}$, and solve problems (34)-(36) and (37) to make the inventory replenishment decisions. We refer to this solution method as BA, standing for balance assumption. Under the relaxation strategy, we solve the optimality equations in (8) and (9) by setting all of the Lagrange multipliers to zero to compute $\left\{v_{i t}^{L}(\cdot \mid 0): i \in \mathcal{I} \cup\{\phi\}, t \in \mathcal{T}\right\}$. Similar to $L R$ and BA, we let $\vartheta_{i t}(\cdot)=v_{i t}^{L}(\cdot \mid 0)$ for all $i \in \mathcal{I} \cup\{\phi\}$ and $t \in \mathcal{T}$ and make the inventory replenishment decisions by solving problems (34)-(36) and (37). We refer to this solution method as RS, standing for relaxation strategy.

\subsection{Computational Results}

Table 1 shows the computational results for the 21 test problems in the base case. Each row in this table corresponds to a particular instance of a demand profile. The first column shows the demand profile and the instance number. The second, third, and fourth columns show the lower bounds on the value function obtained by LR, BA, and RS. The fifth column shows the percent gap between the lower bounds obtained by LR and BA. The sixth column does the same thing as the fifth column, but it focuses on the percent gap between LR and RS. The seventh, eighth, and ninth columns show the expected costs incurred by LR, BA, and RS. We estimate these expected costs by simulating the performance of the inventory replenishment policies obtained by LR, BA, and RS under multiple demand realizations. The 10th column shows the percent gap between the expected costs incurred by LR and BA. We simulate the performance for enough demand realizations, so that any performance gap that exceeds $1 \%$ is statistically significant. We cannot guarantee that smaller performance gaps are statistically significant, because there are some test problems where the benchmarks perform very similarly and making a statistically significant distinction between them requires an exorbitant number of demand realizations. The 11th column does the same thing as the 10th column, but it focuses on the percent gap between LR and RS.

The results indicate that the three benchmarks obtain very similar lower bounds and incur very similar expected costs under demand profile $\mathcal{D}_{1}$. Furthermore, the expected costs are very close to the lower bounds, which indicates that the three benchmarks are essentially optimal under demand profile $\mathcal{D}_{1}$. When we move to demand profiles $\mathcal{D}_{2}$ and $\mathcal{D}_{3}$, the lower bounds and expected costs associated with LR become noticeably better than those associated with BA and RS. For 
Table 2. Effect of the holding cost at the warehouse on the performance of LR, BA, and RS.

\begin{tabular}{crrrrr}
\hline & \multicolumn{2}{c}{$\begin{array}{c}\text { Lower bnd. } \\
\text { LR vs. }\end{array}$} & & \multicolumn{2}{c}{$\begin{array}{c}\text { Exp. cost } \\
\text { LR vs. }\end{array}$} \\
\cline { 2 - 3 } \cline { 5 - 6 } Hld. cst. & BA & RS & & BA & RS \\
\hline $\mathcal{D}_{1}$ & & & & \\
0.1 & -0.02 & 0.00 & & 0.00 & 0.00 \\
0.3 & -0.01 & 0.00 & & 0.00 & 0.00 \\
0.6 & -0.04 & 0.00 & & -0.04 & 0.00 \\
0.9 & 0.00 & 0.00 & & 0.00 & 0.00 \\
$\mathcal{D}_{2}$ & & & & \\
0.1 & -0.57 & 11.26 & & 0.69 & 5.30 \\
0.3 & -0.14 & 7.49 & 1.60 & 5.86 \\
0.6 & 0.70 & 4.38 & & 4.58 & 7.98 \\
0.9 & 2.02 & 2.87 & 7.36 & 8.53 \\
$\mathcal{D}_{3}$ & & & & \\
0.1 & -5.89 & 45.60 & -1.13 & 21.94 \\
0.3 & -2.57 & 32.10 & & 4.43 & 20.60 \\
0.6 & 3.19 & 21.13 & 9.58 & 20.64 \\
0.9 & 10.53 & 15.13 & 16.67 & 23.12 \\
\hline
\end{tabular}

example, the average gap between the lower bounds obtained by LR and BA increases to $3.19 \%$ under demand profile $\mathcal{D}_{3}$. Under the same demand profile, the average gap between the expected costs incurred by LR and BA increases to $9.58 \%$. The performance of RS is quite satisfactory under demand profile $\mathcal{D}_{1}$, but the performance gap between LR and RS can be as large as $25.15 \%$ under demand profile $\mathcal{D}_{3}$. Therefore, the nonstationarity of the demand distributions seems

Table 3. Effect of the backlogging cost at the retailers on the performance of LR, BA, and RS.

\begin{tabular}{crrrrr}
\hline & \multicolumn{2}{c}{$\begin{array}{c}\text { Lower bnd. } \\
\text { LR vs. }\end{array}$} & & \multicolumn{2}{c}{$\begin{array}{c}\text { Exp. cost } \\
\text { LR vs. }\end{array}$} \\
\cline { 2 - 3 } \cline { 5 - 6 } Bck. cst. & BA & RS & & BA & RS \\
\hline $\mathcal{D}_{1}$ & & & & \\
1 & 0.00 & 0.00 & & 0.00 & 0.00 \\
9 & 0.00 & 0.00 & & 0.00 & 0.00 \\
19 & -0.04 & 0.00 & & -0.04 & 0.00 \\
29 & 0.00 & 0.00 & & 0.00 & 0.00 \\
39 & 0.00 & 0.00 & & 0.00 & 0.00 \\
$\mathcal{D}_{2}$ & & & & \\
1 & -1.27 & 0.00 & & -1.10 & 0.05 \\
9 & 0.18 & 3.49 & & 2.07 & 4.84 \\
19 & 0.70 & 4.38 & & 4.58 & 7.98 \\
29 & 0.88 & 4.62 & & 6.51 & 9.43 \\
39 & 0.95 & 4.65 & & 7.92 & 11.69 \\
$\mathcal{D}_{3}$ & & & & \\
1 & -6.20 & 0.03 & & -8.27 & 0.32 \\
9 & -0.92 & 15.67 & 3.02 & 14.80 \\
19 & 3.19 & 21.13 & 9.58 & 20.64 \\
29 & 4.68 & 22.07 & 12.85 & 23.77 \\
39 & 5.19 & 21.75 & 13.26 & 24.38 \\
\hline
\end{tabular}

Table 4. Effect of the number of retailers on the performance of $\mathrm{LR}, \mathrm{BA}$, and RS.

\begin{tabular}{crrrrr}
\hline & \multicolumn{2}{c}{$\begin{array}{c}c \\
\text { LR vs. }\end{array}$} & & \multicolumn{2}{c}{$\begin{array}{c}\text { Exp. cost } \\
\text { LR vs. }\end{array}$} \\
\cline { 2 - 3 } \cline { 5 - 6 } No. ret. & BA & RS & & BA & RS \\
\hline $\mathcal{D}_{1}$ & & & & \\
1 & 0.00 & 0.00 & & 0.00 & 0.00 \\
2 & 0.00 & 0.00 & & 0.00 & 0.00 \\
3 & -0.04 & 0.00 & & -0.04 & 0.00 \\
4 & 0.00 & 0.00 & & 0.00 & 0.00 \\
5 & 0.00 & 0.00 & & 0.00 & 0.00 \\
$\mathcal{D}_{2}$ & & & & \\
1 & -0.16 & 3.61 & & -0.02 & 0.24 \\
2 & 0.37 & 3.96 & & 2.54 & 6.71 \\
3 & 0.70 & 4.38 & & 4.58 & 7.98 \\
4 & 1.10 & 4.99 & & 5.60 & 8.38 \\
5 & 1.46 & 5.48 & & 6.18 & 8.93 \\
$\mathcal{D}_{3}$ & & & & \\
1 & -0.17 & 0.03 & & -0.07 & 0.00 \\
2 & 0.63 & 9.93 & 3.75 & 12.50 \\
3 & 3.19 & 21.13 & 9.58 & 20.64 \\
4 & 2.55 & 27.46 & 9.91 & 25.53 \\
5 & 2.25 & 33.09 & 9.09 & 24.53 \\
\hline
\end{tabular}

to have a large impact on the performance of the benchmarks, and LR tends to perform better than BA and RS when the demand distributions involve significant nonstationarity. Another important observation is that there are test problems where BA performs better, but the performance gap is relatively small when this is the case. Furthermore, the large performance gap between LR and RS shows the importance of finding a good set of values for the Lagrange multipliers

Table 5. Effect of the length of the planning horizon on the performance of LR, BA, and RS.

\begin{tabular}{crrrrr}
\hline & \multicolumn{2}{c}{$\begin{array}{c}\text { Lower bnd. } \\
\text { LR vs. }\end{array}$} & & \multicolumn{2}{c}{$\begin{array}{c}\text { Exp. cost } \\
\text { LR vs. }\end{array}$} \\
\cline { 2 - 3 } \cline { 5 - 6 } P1. hor. & BA & RS & & BA & RS \\
\hline $\mathcal{D}_{1}$ & & & & \\
25 & 0.00 & 0.00 & & 0.00 & -0.34 \\
50 & -0.04 & 0.00 & & -0.04 & 0.00 \\
75 & 0.00 & 0.00 & & 0.00 & 0.00 \\
100 & 0.00 & 0.00 & & 0.00 & 0.00 \\
$\mathcal{D}_{2}$ & & & & \\
25 & 0.82 & 4.32 & & 5.21 & 9.07 \\
50 & 0.70 & 4.38 & & 4.58 & 7.98 \\
75 & 0.80 & 4.92 & & 5.72 & 9.55 \\
100 & 0.83 & 4.95 & 5.93 & 9.74 \\
$\mathcal{D}_{3}$ & & & & \\
25 & 2.10 & 15.96 & 9.46 & 21.80 \\
50 & 3.19 & 21.13 & 9.58 & 20.64 \\
75 & 3.69 & 23.70 & 9.26 & 23.42 \\
100 & 3.49 & 24.07 & 9.25 & 22.34 \\
\hline
\end{tabular}

Naval Research Logistics DOI 10.1002/nav 
Table 6. Effect of the lead time for the warehouse on the performance of LR, BA, and RS.

\begin{tabular}{crrrrr}
\hline & \multicolumn{2}{c}{$\begin{array}{c}\text { Lower bnd. } \\
\text { LR vs. }\end{array}$} & & \multicolumn{2}{c}{$\begin{array}{c}\text { Exp. cost } \\
\text { LR vs. }\end{array}$} \\
\cline { 2 - 3 } \cline { 5 - 6 } Wh. 1.t. & BA & RS & & BA & RS \\
\hline $\mathcal{D}_{1}$ & & & & \\
1 & -0.04 & 0.00 & & -0.04 & 0.00 \\
2 & 0.00 & 0.00 & & 0.00 & 0.00 \\
3 & 0.00 & 0.00 & & 0.00 & 0.00 \\
$\mathcal{D}_{2}$ & & & & \\
1 & 0.70 & 4.38 & & 4.58 & 7.98 \\
2 & -0.02 & 3.32 & & 0.84 & 3.15 \\
3 & -0.13 & 2.90 & & 0.23 & 1.98 \\
$\mathcal{D}_{3}$ & & & & \\
1 & 3.19 & 21.13 & & 9.58 & 20.64 \\
2 & -0.05 & 15.62 & & 4.70 & 20.17 \\
3 & -1.21 & 12.99 & 1.38 & 19.26 \\
\hline
\end{tabular}

by solving problem (17) rather than using the trivial value of zero.

Tables 2-7 show how the benchmarks perform when we vary different problem parameters. We begin by varying the holding cost at the warehouse in Table 2 . For economy of space, we only show the average results over seven instances of a particular demand profile. More specifically, the three portions of Table 2 , respectively, focus on demand profiles $\mathcal{D}_{1}, \mathcal{D}_{2}$, and $\mathcal{D}_{3}$. In each portion, the first column shows the value of the holding cost at the warehouse. The second column shows the percent gap between the lower bounds on the value function obtained by LR and BA, averaged over seven instances of a particular demand profile. The fourth columns show the percent gap between the expected costs incurred by LR and BA, averaged over seven instances of a particular demand profile. The third and fifth columns do the same thing as the second and fourth columns, but they focus on the percent gap between LR and RS. It is helpful to note that the percent gaps are calculated in such a manner that positive values favor LR and negative values favor the other two benchmarks. We also emphasize that averaging does not change the interpretation of the results significantly. In particular, if the average performance of a particular benchmark is noticeably better than that of another benchmark, then the performance is generally better over all seven instances.

Table 2 indicates that the benchmarks continue to perform similarly under demand profile $\mathcal{D}_{1}$. Under demand profiles $\mathcal{D}_{2}$ and $\mathcal{D}_{3}$, the average gap between the lower bounds obtained by LR and BA increases in favor of LR as the holding cost at the warehouse increases. A similar observation also applies to the average gap between the expected costs incurred by LR and BA. The average gap between the lower bounds obtained by LR and RS decreases as the holding cost at the warehouse increases, but there is no drastic change in the average gap between the expected costs incurred by LR and RS.

In Table 3, we vary the backlogging cost at the retailers. The observations for demand profile $\mathcal{D}_{1}$ remain unchanged. Under demand profiles $\mathcal{D}_{2}$ and $\mathcal{D}_{3}$, BA obtains tighter lower bounds and incurs lower expected costs than LR when the backlogging cost at the retailers is low. Nevertheless, we note that a backlogging cost of one is quite low as the holding cost at the retailers is also one. As the backlogging cost at the retailers increases, the lower bounds and expected costs associated with LR become significantly better than those associated with BA. The performance gap between LR and RS also increases in favor of LR as the backlogging cost at the retailers increases. Dogru [9] also mentions backlogging cost at the retailers as a factor that affects the adequacy of the balance assumption.

Table 4 varies the number of retailers in the distribution system. Under demand profiles $\mathcal{D}_{2}$ and $\mathcal{D}_{3}$, the average gap between the expected costs incurred by LR and BA increases as the number of retailers increases. For distribution systems with five retailers, the average gap between the expected costs incurred by LR and BA is as large as $9.09 \%$. Clark and Scarf [8] show that BA obtains the optimal policy when there is one retailer. Although LR does not have such an optimality guarantee, its performance for the test problems with one retailer is very close to optimal. The results are quite encouraging in the sense that LR tends to perform significantly better than BA and maintains its performance margin when we work with larger distribution systems. Except for the test problems with one retailer, the performance gap between LR and RS is also quite significant.

In Table 5, we vary the length of the planning horizon. There does not seem to be a drastic effect of the length of the planning horizon on the relative performance of the benchmarks.

Table 7. Effect of the lead time for the retailers on the performance of LR, BA, and RS.

\begin{tabular}{crrrrr}
\hline & \multicolumn{2}{c}{$\begin{array}{c}\text { Lower bnd. } \\
\text { LR vs. }\end{array}$} & & \multicolumn{2}{c}{$\begin{array}{c}\text { Exp. cost } \\
\text { LR vs. }\end{array}$} \\
\cline { 2 - 3 } \cline { 5 - 6 } Ret. 1.t. & BA & RS & & BA & RS \\
\hline $\mathcal{D}_{1}$ & & & & \\
1 & -0.04 & 0.00 & & -0.04 & 0.00 \\
2 & 0.00 & 0.00 & & 0.00 & 0.00 \\
3 & 0.00 & 0.00 & & 0.00 & 0.00 \\
$\mathcal{D}_{2}$ & & & & \\
1 & 0.70 & 4.38 & & 4.58 & 7.98 \\
2 & 0.20 & 1.23 & & 2.03 & 3.39 \\
3 & 0.05 & 0.47 & & 0.92 & 1.60 \\
$\mathcal{D}_{3}$ & & & & \\
1 & 3.19 & 21.13 & & 9.58 & 20.64 \\
2 & 2.80 & 13.02 & 6.77 & 12.87 \\
3 & 1.45 & 7.63 & 5.09 & 10.74 \\
\hline
\end{tabular}


Table 6 varies the lead time for the replenishments of the warehouse, and Table 7 varies the lead time for the replenishments of the retailers. As the lead times increase, BA becomes comparable to LR. RS consistently lags behind LR and BA.

All the computational experiments were carried out in MATLAB 7.0 on a Pentium D Desktop PC with $3.4 \mathrm{GHz}$ CPU and 1 GB RAM running Windows XP. For given Lagrange multipliers, the CPU time required to compute $\left\{V_{t}^{L}(\cdot \mid \lambda): t \in \mathcal{T}\right\}$ is comparable to the CPU time required to compute $\left\{V_{t}^{B}(\cdot): t \in \mathcal{T}\right\}$. This is not surprising, because the optimality equations in (8) and (9) are similar to those in (23) and (25). Therefore, BA and RS have comparable CPU times. On the other hand, because LR solves problem (17) by using subgradient optimization, the CPU time for LR is considerably longer than the CPU time for BA. Table 8 shows the CPU times for LR and BA for the 21 test problems in the base case and indicates that the CPU time for LR is longer than the CPU time for BA by orders of magnitude. Nevertheless, we emphasize that problem (17) is solved in an offline manner, and long CPU times for LR should not interfere with its practical appeal. Once we obtain the optimal solution to problem (17), the CPU time required to make the inventory replenishment decisions is essentially the same for LR and BA, because both LR and BA make the inventory replenishment decisions by solving problems (34)-(36) and (37). Finally, Fig. 2 plots the CPU times for LR and BA as a function of the length of the planning horizon and the number
Table 8. CPU times for LR and BA for the 21 test problems in the base case.

\begin{tabular}{llc}
\hline & \multicolumn{2}{c}{ CPU (sec.) } \\
\cline { 2 - 3 } Prob. & LR & BA \\
\hline$\left(\mathcal{D}_{1}, 1\right)$ & 351 & 0.50 \\
$\left(\mathcal{D}_{1}, 2\right)$ & 357 & 0.47 \\
$\left(\mathcal{D}_{1}, 3\right)$ & 361 & 0.48 \\
$\left(\mathcal{D}_{1}, 4\right)$ & 368 & 0.51 \\
$\left(\mathcal{D}_{1}, 5\right)$ & 375 & 0.55 \\
$\left(\mathcal{D}_{1}, 6\right)$ & 362 & 0.41 \\
$\left(\mathcal{D}_{1}, 7\right)$ & 369 & 0.44 \\
$\left(\mathcal{D}_{2}, 1\right)$ & 491 & 0.73 \\
$\left(\mathcal{D}_{2}, 2\right)$ & 495 & 0.52 \\
$\left(\mathcal{D}_{2}, 3\right)$ & 479 & 0.50 \\
$\left(\mathcal{D}_{2}, 4\right)$ & 496 & 0.52 \\
$\left(\mathcal{D}_{2}, 5\right)$ & 510 & 0.53 \\
$\left(\mathcal{D}_{2}, 6\right)$ & 513 & 0.63 \\
$\left(\mathcal{D}_{2}, 7\right)$ & 507 & 0.72 \\
$\left(\mathcal{D}_{3}, 1\right)$ & 0.54 \\
$\left(\mathcal{D}_{3}, 2\right)$ & 376 & 0.42 \\
$\left(\mathcal{D}_{3}, 3\right)$ & 344 & 0.39 \\
$\left(\mathcal{D}_{3}, 4\right)$ & 350 & 0.52 \\
$\left(\mathcal{D}_{3}, 5\right)$ & 353 & 0.49 \\
$\left(\mathcal{D}_{3}, 6\right)$ & 353 & 0.45 \\
$\left(\mathcal{D}_{3}, 7\right)$ & 359 & 0.40 \\
\hline & 351 &
\end{tabular}

of retailers. This figure indicates that the CPU times for both LR and BA scale approximately linearly with the size of the problem.
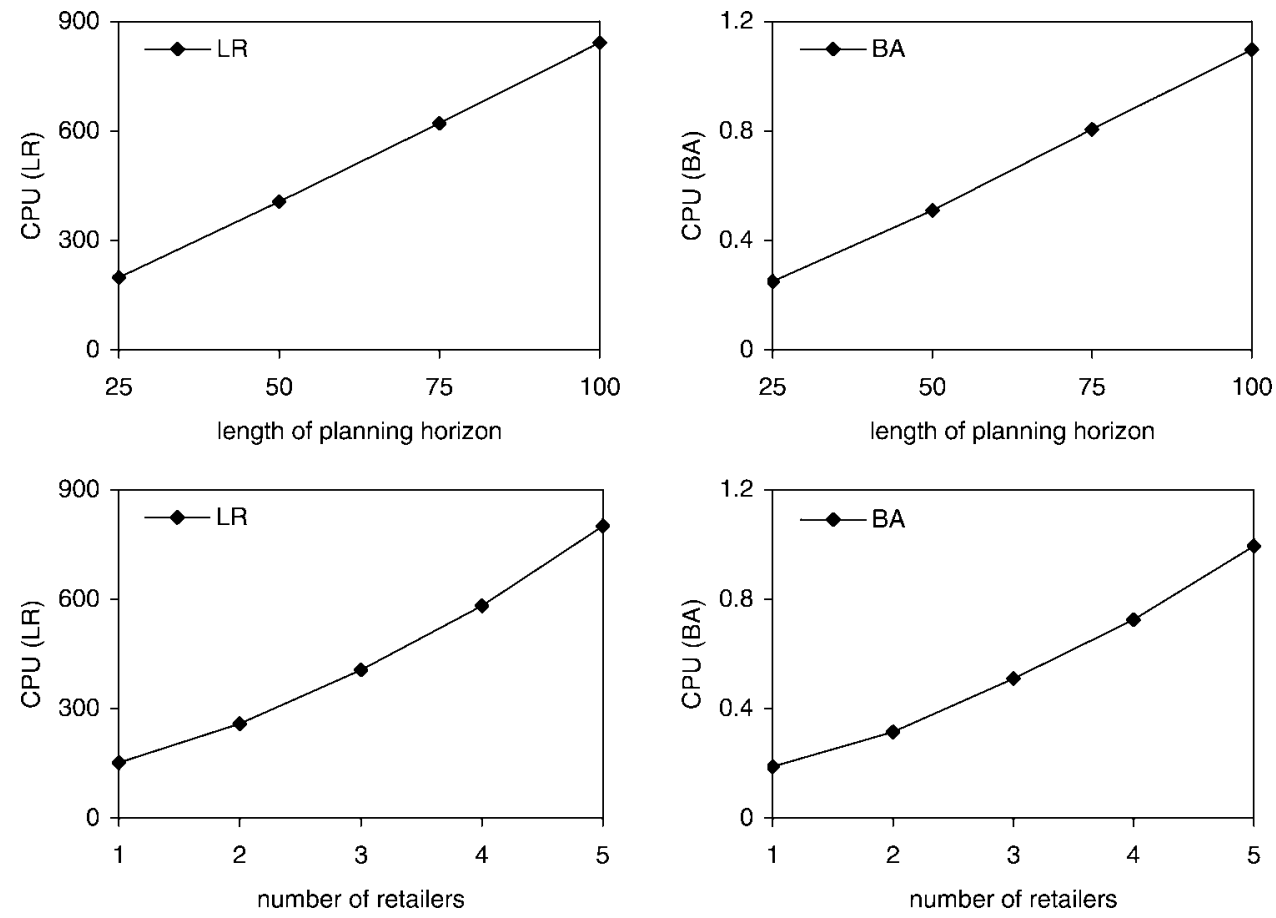

Figure 2. CPU times for LR and BA as a function of the length of the planning horizon and the number of retailers. 


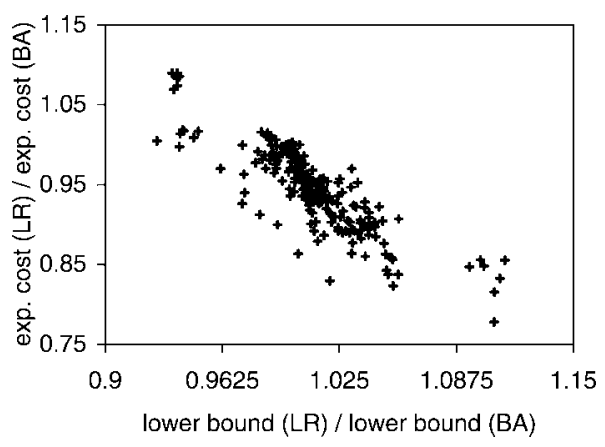

Figure 3. The pairs $\left[V_{1}^{L}\left(x_{1} \mid \hat{\lambda}\right) / V_{1}^{B}\left(x_{1}\right), \Pi^{L R} / \Pi^{B A}\right]$ for all of the 400 test problems in our experimental setup.

In summary, BA and RS perform well under relatively stationary demand distributions. Indeed, BA and RS perform as well as LR for all of our test problems under demand profile $\mathcal{D}_{1}$. On the other hand, LR has the potential to provide advantages when the demand distributions involve significant nonstationarity. In addition to the nonstationarity of the demand distributions, high holding cost at the warehouse, high backlogging cost at the retailers, and large number of retailers in the distribution system emerge as other factors that may boost the performance of LR relative to BA and RS. Another interesting observation from our computational experiments is that the gap between the expected costs tends to be significantly larger than the gap between the lower bounds. This is worth mentioning because the policy performance is more relevant than the quality of the lower bounds from a practical perspective. Finally, we note that the gap between the lower bounds on the value function obtained by LR and BA seems to be a good predictor of when LR performs better than BA. In particular, letting $\hat{\lambda}$ be the optimal solution to problem (17), and $\Pi^{L R}$ and $\Pi^{B A}$ respectively be the expected costs incurred by LR and BA, Fig. 3 plots the pairs $\left[V_{1}^{L}\left(x_{1} \mid \hat{\lambda}\right) / V_{1}^{B}\left(x_{1}\right), \Pi^{L R} / \Pi^{B A}\right]$ for all of the 400 test problems in our experimental setup. This figure indicates that whenever LR obtains a significantly tighter lower bound than BA, it also incurs a significantly lower cost than BA.

\section{CONCLUSIONS}

In this work, we developed a new method for making the inventory replenishment decisions in a distribution system. Our method is based on formulating the problem as a dynamic program and using Lagrange multipliers to relax the constraints that ensure the non-negativity of the shipments to the retailers. Because the relaxation strategy of Federgruen and Zipkin [14] is a special case of our method that is obtained by setting all of the Lagrange multipliers to zero, our method naturally improves the lower bound on the value function that is obtained under the relaxation strategy. Computational experiments indicate that we can tighten the lower bounds on the value functions and improve the performance of the inventory replenishment policies significantly by choosing a good set of values for the Lagrange multipliers. Although our method does not always perform better than the inventory replenishment policies obtained under the balance assumption of Clark and Scarf [8], it appears to be a viable alternative when the balance assumption remains inadequate. From our computational experiments, a distribution system with highly nonstationary demand distributions, high holding cost at the warehouse, high backlogging cost at the retailers, and large number of retailers appears to be an ideal candidate for applying our method. Furthermore, the gap between the lower bounds on the value function obtained by our method and the balance assumption is a good predictor of when our method would perform better than the balance assumption.

\section{APPENDIX A: OMITTED RESULTS IN THE PAPER}

Proof of Proposition 5 uses the next lemma.

LEMMA 7: If $x_{\phi t}<\sum_{i \in \mathcal{I}} \hat{r}_{i t}$ and the optimal solution to the problem

$$
\begin{aligned}
\min & \sum_{i \in \mathcal{I} \cup\{\phi\}} c_{i t}\left[y_{i t}-x_{i t}\right]+\sum_{i \in \mathcal{I} \cup\{\phi\}} L_{i t}\left(y_{i t}\right) \\
& +\sum_{i \in \mathcal{I} \cup\{\phi\}} \mathbb{E}\left\{v_{i, t+1}^{B}\left(y_{i t}-D_{i t}\right)\right\} \\
\text { subject to } & \text { (2), (3) }
\end{aligned}
$$

is the same as the optimal solution to problem (28)-(30), then we have $v_{i t}^{B}\left(x_{i t}\right)=c_{i t}\left[\hat{r}_{i t}-x_{i t}\right]+L_{i t}\left(\hat{r}_{i t}\right)+\mathbb{E}\left\{v_{i, t+1}^{B}\left(\hat{r}_{i t}-D_{i t}\right)\right\}$ for all $i \in \mathcal{I}$.

PROOF: For all $i \in \mathcal{I}$, we let $\tilde{r}_{i t}$ be the largest optimal solution to problem (24). If we can show that $\tilde{r}_{i t} \geq x_{i t}$ for all $i \in \mathcal{I}$, then $\tilde{r}_{i t}$ is a feasible solution to problem (23) for all $i \in \mathcal{I}$ and the result follows. To obtain a contradiction, we assume that $y_{t}^{1}=\left\{y_{i t}^{1}: i \in \mathcal{I} \cup\{\phi\}\right\}$ is the common optimal solution to problem (28)-(30) and (38)-(39), but we have $\tilde{r}_{i^{\prime} t}<x_{i^{\prime} t}$ for some $i^{\prime} \in \mathcal{I}$. The solution $y_{t}^{2}=\left\{y_{i t}^{2}: i \in \mathcal{I} \cup\{\phi\}\right\}$ obtained by letting $y_{i t}^{2}=y_{i t}^{1}$ for all $i \in \mathcal{I} \backslash\left\{i^{\prime}\right\}, y_{i^{\prime} t}^{2}=\tilde{r}_{i^{\prime} t}$ and $y_{\phi t}^{2}=y_{\phi t}^{1}$ is feasible to problem (28)-(30). Because the solution $y_{t}^{1}$ satisfies constraints (3), we have $\tilde{r}_{i^{\prime} t}<x_{i^{\prime} t} \leq y_{i^{\prime} t}^{1}$. Therefore, because $\tilde{r}_{i^{\prime} t}$ is the largest minimizer of the function $c_{i^{\prime} t}\left[y_{i^{\prime} t}-x_{i^{\prime} t}\right]+L_{i^{\prime} t}\left(y_{i^{\prime} t}\right)+\mathbb{E}\left\{v_{i^{\prime}, t+1}^{B}\left(y_{i^{\prime} t}-D_{i^{\prime} t}\right)\right\}$, the objective value obtained by the solution $y_{t}^{2}$ for problem (28)-(30) is strictly less than the objective value obtained by the solution $y_{t}^{1}$. This contradicts the fact that $y_{t}^{1}$ is the optimal solution to problem (28)-(30).

Proof of Proposition 6 uses the next lemma.

LEMMA 8: The optimal objective value of the problem

$$
\min \sum_{i \in \mathcal{I}} c_{i t}\left[y_{i t}-x_{i t}\right]+\sum_{i \in \mathcal{I}} L_{i t}\left(y_{i t}\right)+\sum_{i \in \mathcal{I}} \mathbb{E}\left\{v_{i, t+1}^{B}\left(y_{i t}-D_{i t}\right)\right\}
$$

$$
\begin{array}{ll}
\text { subject to } & \sum_{i \in \mathcal{I}} y_{i t} \leq x_{\phi t} \\
& y_{i t} \geq x_{i t} \quad \text { for all } i \in \mathcal{I}
\end{array}
$$


is bounded from below by

$$
\begin{aligned}
& \Delta_{t}^{B}\left(x_{\phi t}\right)+\sum_{i \in \mathcal{I}} \min _{y_{i t} \geq x_{i t}}\left\{c_{i t}\left[y_{i t}-x_{i t}\right]+L_{i t}\left(y_{i t}\right)\right. \\
&\left.+\mathbb{E}\left\{v_{i, t+1}^{B}\left(y_{i t}-D_{i t}\right)\right\}\right\} .
\end{aligned}
$$

PROOF: We let $\tilde{y}_{t}=\left\{\tilde{y}_{i t}: i \in \mathcal{I}\right\}$ be the optimal solution to problem (40)-(42). We define the new solution $\hat{y}_{t}=\left\{\hat{y}_{i t}: i \in \mathcal{I}\right\}$ as $\hat{y}_{i t}=\tilde{y}_{i t}$ if $\tilde{y}_{i t} \leq \hat{r}_{i t}$ and $\hat{y}_{i t}=\hat{r}_{i t}$ if $\tilde{y}_{i t}>\hat{r}_{i t}$. Noting that $\sum_{i \in \mathcal{I}} \tilde{y}_{i t} \leq x_{\phi t}$ and $\tilde{y}_{i t} \geq \hat{y}_{i t}$ for all $i \in \mathcal{I}$, the solution $\hat{y}_{t}$ is feasible to problem (26)-(27). Evaluating the objective function of problem (26)-(27) at the feasible solution $\hat{y}_{t}$, we have

$$
\begin{array}{rl}
\Delta_{t}^{B}\left(x_{\phi t}\right) \leq \sum_{i \in \mathcal{I}} & \mathbf{1}\left(\tilde{y}_{i t} \leq \hat{r}_{i t}\right)\left\{c_{i t}\left[\tilde{y}_{i t}-\hat{r}_{i t}\right]+L_{i t}\left(\tilde{y}_{i t}\right)\right. \\
& \left.+\mathbb{E}\left\{v_{i, t+1}^{B}\left(\tilde{y}_{i t}-D_{i t}\right)\right\}-L_{i t}\left(\hat{r}_{i t}\right)-\mathbb{E}\left\{v_{i, t+1}^{B}\left(\hat{r}_{i t}-D_{i t}\right)\right\}\right\},
\end{array}
$$

where $\mathbf{1}(\cdot)$ is the indicator function. On the other hand, because $\tilde{y}_{i t} \geq x_{i t}$ for all $i \in \mathcal{I}$, we have

$$
\begin{aligned}
\min _{y_{i t} \geq x_{i t}} & \left\{c_{i t}\left[y_{i t}-x_{i t}\right]+L_{i t}\left(y_{i t}\right)+\mathbb{E}\left\{v_{i, t+1}^{B}\left(y_{i t}-D_{i t}\right)\right\}\right\} \\
\leq & \mathbf{1}\left(\tilde{y}_{i t} \leq \hat{r}_{i t}\right)\left\{c_{i t}\left[\hat{r}_{i t}-x_{i t}\right]+L_{i t}\left(\hat{r}_{i t}\right)+\mathbb{E}\left\{v_{i, t+1}^{B}\left(\hat{r}_{i t}-D_{i t}\right)\right\}\right\} \\
& \quad+\mathbf{1}\left(\tilde{y}_{i t}>\hat{r}_{i t}\right)\left\{c_{i t}\left[\tilde{y}_{i t}-x_{i t}\right]+L_{i t}\left(\tilde{y}_{i t}\right)+\mathbb{E}\left\{v_{i, t+1}^{B}\left(\tilde{y}_{i t}-D_{i t}\right)\right\}\right\}
\end{aligned}
$$

for all $i \in \mathcal{I}$. Adding the last two inequalities, it is easy to see that the expression in (43) provides a lower bound on $\sum_{i \in \mathcal{I}} c_{i t}\left[\tilde{y}_{i t}-x_{i t}\right]+\sum_{i \in \mathcal{I}} L_{i t}\left(\tilde{y}_{i t}\right)+$ $\sum_{i \in \mathcal{I}} \mathbb{E}\left\{v_{i, t+1}^{B}\left(\tilde{y}_{i t}-D_{i t}\right)\right\}$, which is the optimal objective value of problem (40)-(42).

\section{APPENDIX B: EXAMPLES THAT COMPARE THE LOWER BOUNDS ON THE VALUE FUNCTIONS}

In this section, we present two examples to show that we can have $\max _{\lambda \geq 0}\left\{V_{1}^{L}\left(x_{1} \mid \lambda\right)\right\}>V_{1}^{B}\left(x_{1}\right)$ or $\max _{\lambda \geq 0}\left\{V_{1}^{L}\left(x_{1} \mid \lambda\right)\right\}<V_{1}^{B}\left(x_{1}\right)$ depending on the problem parameters. Therefore, there does not exist a consistent ordering between the lower bounds computed under the Lagrangian relaxation strategy and the balance assumption.

\section{An Example with $\max _{\lambda \geq 0}\left\{V_{1}^{L}\left(x_{1} \mid \lambda\right)\right\}>V_{I}^{B}\left(x_{1}\right)$}

We consider a problem instance with $\mathcal{I}=\{a, b\}, \mathcal{T}=\{1\}, h_{a 1}=h_{b 1}=$ $10, h_{\phi 1}=5, b_{a 1}=b_{b 1}=10$, and $c_{a 1}=c_{b 1}=c_{\phi 1}=0$. For the demand random variables, we have $D_{a 1}=10$ and $D_{b 1}=5$ with probability 1 . The initial state variable is given by $x_{a 1}=5, x_{b 1}=10$, and $x_{\phi 1}=15$. We show that $\max _{\lambda \geq 0}\left\{V_{1}^{L}\left(x_{1} \mid \lambda\right)\right\}>V_{1}^{B}\left(x_{1}\right)$ for this problem instance.

\section{Lagrangian Relaxation Strategy}

We first consider the Lagrangian relaxation strategy. We let $\hat{\lambda}_{a 1}=0$ and $\hat{\lambda}_{b 1}=20$, in which case problem (4)-(6) imply that

$$
\begin{aligned}
V_{1}^{L}\left(x_{1} \mid \hat{\lambda}\right)=\min \quad & 5 y_{\phi 1}-20\left[y_{b 1}-10\right]-5 y_{a 1}+10\left[y_{a 1}-10\right]^{+} \\
& +10\left[10-y_{a 1}\right]^{+}-5 y_{b 1}+10\left[y_{b 1}-5\right]^{+}+10\left[5-y_{b 1}\right]^{+} \\
\text {subject to } & y_{a 1}+y_{b 1} \leq 15 \\
& y_{\phi 1} \geq 15 .
\end{aligned}
$$

It is easy to check that $y_{a 1}=0, y_{b 1}=15$, and $y_{\phi 1}=15$ is the optimal solution to the problem above, and this solution yields the optimal objective value of 100 . Therefore, we have $\max _{\lambda \geq 0}\left\{V_{1}^{L}\left(x_{1} \mid \lambda\right)\right\} \geq V_{1}^{L}\left(x_{1} \mid \hat{\lambda}\right)=100$.

\section{Clark and Scarf's Balance Assumption}

We consider the balance assumption next. By (23), we have

$$
v_{a 1}^{B}(5)=\min _{y_{a 1} \geq 5}\left\{-5 y_{a 1}+10\left[y_{a 1}-10\right]^{+}+10\left[10-y_{a 1}\right]^{+}\right\}=-50 .
$$

The unconstrained minimizer of the objective function of the problem above is 10 , so that $\hat{r}_{a 1}=10$. Similarly, we have

$$
v_{b 1}^{B}(10)=\min _{y_{b 1} \geq 10}\left\{-5 y_{b 1}+10\left[y_{b 1}-5\right]^{+}+10\left[5-y_{b 1}\right]^{+}\right\}=0
$$

and $\hat{r}_{b 1}=5$. Noting the argument in Case 1 in the proof of Proposition 5 and the fact that $15 \geq \hat{r}_{a 1}+\hat{r}_{b 1}$, we have $\Delta_{1}^{B}(15)=0$. By (25), we have

$$
v_{\phi 1}^{B}(15)=\min _{y_{\phi 1} \geq 15}\left\{5 y_{\phi 1}\right\}=75 .
$$

Therefore, we obtain $V_{1}^{B}\left(x_{1}\right)=v_{a 1}^{B}(5)+v_{b 1}^{B}(10)+v_{\phi 1}^{B}(15)=25<100 \leq$ $\max _{\lambda \geq 0}\left\{V_{1}^{L}\left(x_{1} \mid \lambda\right)\right\}$.

Because the demands are deterministic, the Lagrangian relaxation strategy for this problem instance is equivalent to the standard Lagrangian relaxation for deterministic optimization problems. This implies that the lower bound computed under the Lagrangian relaxation strategy is tight and we have $\max _{\lambda \geq 0}\left\{V_{1}^{L}\left(x_{1} \mid \lambda\right)\right\}=V_{1}\left(x_{1}\right)$. On the other hand, noting that $y_{a 1}=10$ and $y_{b 1}=5$ is the only feasible solution that satisfies the constraints $y_{a 1}+y_{b 1} \leq 15, y_{a 1} \geq 10$, and $y_{b 1} \geq 5$, it is easy to check that the optimal objective value of problem (1)-(3) for the first time period is 100 . If we drop the constraint $y_{a 1}+y_{b 1} \leq 15$ from problem (1)-(3), then the optimal objective value of problem (1)-(3) decreases to 25 . Therefore, the constraint $y_{a 1}+y_{b 1} \leq 15$ is not redundant in problem (1)-(3), and because we have $x_{\phi 1} \geq \hat{r}_{a 1}+\hat{r}_{b 1}$, (A.1) in the balance assumption does not hold. This implies that Proposition 5 does not apply, and we do not necessarily have $V_{1}\left(x_{1}\right)=V_{1}^{B}\left(x_{1}\right)$. We only have $V_{1}\left(x_{1}\right) \geq V_{1}^{B}\left(x_{1}\right)$ by Proposition 6, but the inequality turns out to be strict for this problem instance.

\section{An Example with $\max _{\lambda \geq 0}\left\{V_{I}^{L}\left(x_{1} \mid \lambda\right)\right\}<V_{I}^{B}\left(x_{1}\right)$}

We consider a problem instance with $\mathcal{I}=\{a, b\}, \mathcal{T}=\{1,2\}, h_{a 1}=$ $h_{b 1}=h_{a 2}=h_{b 2}=10, h_{\phi 1}=h_{\phi 2}=5, b_{a 1}=b_{b 1}=b_{a 2}=b_{b 2}=10$, $c_{a 1}=c_{b 1}=c_{\phi 1}=1$, and $c_{a 2}=c_{b 2}=c_{\phi 2}=0$. For the demand random variables, we have $D_{a 1}=0$ with probability 0.5 and $D_{a 1}=15$ with probability 0.5 , and $D_{b 1}=10, D_{a 2}=10$ and $D_{b 2}=10$ with probability 1 . The initial state variable is given by $x_{a 1}=15, x_{b 1}=21$, and $x_{\phi 1}=75$. We show that $\max _{\lambda \geq 0}\left\{V_{1}^{L}\left(x_{1} \mid \lambda\right)\right\}<V_{1}^{B}\left(x_{1}\right)$ for this problem instance.

\section{Lagrangian Relaxation Strategy}

We first consider the Lagrangian relaxation strategy. By (8), we have

$v_{a 2}^{L}\left(x_{a 2} \mid \lambda\right)=\min _{y_{a 2}}\left\{-\lambda_{a 2}\left[y_{a 2}-x_{a 2}\right]-5 y_{a 2}+10\left[y_{a 2}-10\right]^{+}+10\left[10-y_{a 2}\right]^{+}\right\}$.

It is easy to check that the optimal objective value of the problem above is

$$
v_{a 2}^{L}\left(x_{a 2} \mid \lambda\right)= \begin{cases}-\lambda_{a 2}\left[10-x_{a 2}\right]-50 & \text { if }-15 \leq \lambda_{a 2} \leq 5 \\ -\infty & \text { otherwise, }\end{cases}
$$

and the optimal solution is 10 when $-15 \leq \lambda_{a 2} \leq 5$. Because we are interested in solving the problem $\max _{\lambda \geq 0}\left\{V_{1}^{L}\left(x_{1} \mid \lambda\right)\right\}$, we only focus on the case where $-15 \leq \lambda_{a 2} \leq 5$, which implies that $\hat{r}_{a 2}^{\lambda}=10$.

Because the problem parameters for retailer $b$ at the second time period are the same as those for retailer $a, v_{b 2}^{L}\left(x_{b 2} \mid \lambda\right)$ has the same form as (44), and we have $\hat{r}_{b 2}^{\lambda}=10$ when $-15 \leq \lambda_{b 2} \leq 5$. 
Using the same argument in Case 1 in the proof of Proposition 5, it is easy to see that if $x_{\phi t} \geq \sum_{i \in \mathcal{I}} \hat{r}_{i t}^{\lambda}$, then we have $\Delta_{t}^{L}\left(x_{\phi t} \mid \lambda\right)=0$. In particular, the unconstrained minimizer of the objective function of problem (10)-(11) is $\left\{\hat{r}_{i t}^{\lambda}: i \in \mathcal{I}\right\}$. Furthermore, the solution $\left\{\hat{r}_{i t}^{\lambda}: i \in \mathcal{I}\right\}$ is feasible to problem (10)-(11) when we have $x_{\phi t} \geq \sum_{i \in \mathcal{I}} \hat{r}_{i t}^{\lambda}$. Therefore, the optimal solution to problem (10)-(11) is $\left\{\hat{r}_{i t}^{\lambda}: i \in \mathcal{I}\right\}$, and we have $\Delta_{t}^{L}\left(x_{\phi t} \mid \lambda\right)=0$.

Because, we have $x_{\phi 2}=x_{\phi 1}+q_{\phi 1}-D_{a 1}-D_{b 1} \geq x_{\phi 1}-D_{a 1}-D_{b 1} \geq$ $75-15-10 \geq 20=\hat{r}_{a 2}^{\lambda}+\hat{r}_{b 2}^{\lambda}$ with probability 1 , we can assume that $\Delta_{2}^{L}\left(x_{\phi 2} \mid \lambda\right)=0$ throughout. In this case, by (9), we have

$$
v_{\phi 2}^{L}\left(x_{\phi 2} \mid \lambda\right)=\min _{y_{\phi 2} \geq x_{\phi 2}}\left\{5 y_{\phi 2}\right\}=5 x_{\phi 2} .
$$

Using (8) and (44), we have

$$
\begin{aligned}
& v_{a 1}^{L}(15 \mid \lambda)=\min _{y_{a 1}}\left\{\left[1-\lambda_{a 1}\right]\left[y_{a 1}-15\right]-5 y_{a 1}\right. \\
& \quad+\frac{1}{2}\left\{10\left[y_{a 1}-0\right]^{+}+10\left[0-y_{a 1}\right]^{+}-\lambda_{a 2}\left[10-\left(y_{a 1}-0\right)\right]-50\right\} \\
& \left.+\frac{1}{2}\left\{10\left[y_{a 1}-15\right]^{+}+10\left[15-y_{a 1}\right]^{+}-\lambda_{a 2}\left[10-\left(y_{a 1}-15\right)\right]-50\right\}\right\}
\end{aligned}
$$

One can check that the optimal objective value of the problem above is

$$
v_{a 1}^{L}(15 \mid \lambda)= \begin{cases}-2.5 \lambda_{a 2}-50 & \text { if }-6 \leq-\lambda_{a 1}+\lambda_{a 2} \leq 4 \\ 15 \lambda_{a 1}-17.5 \lambda_{a 2}+10 & \text { if } 4 \leq-\lambda_{a 1}+\lambda_{a 2} \leq 14 \\ -\infty & \text { otherwise }\end{cases}
$$

and the optimal solution is 15 when $-6 \leq-\lambda_{a 1}+\lambda_{a 2} \leq 4$, and 0 when $4 \leq-\lambda_{a 1}+\lambda_{a 2} \leq 14$. We note that there are multiple optimal solutions when $-\lambda_{a 1}+\lambda_{a 2}=4$. Therefore, we have $\hat{r}_{a 1}^{\lambda}=15$ when $-6 \leq-\lambda_{a 1}+\lambda_{a 2} \leq 4$ and $\hat{r}_{a 1}^{\lambda}=0$ when $4 \leq-\lambda_{a 1}+\lambda_{a 2} \leq 14$.

Similarly, we have

$$
\begin{aligned}
v_{b 1}^{L}(21 \mid \lambda)=\min _{y_{b 1}}\left\{\left[1-\lambda_{b 1}\right]\right. & {\left[y_{b 1}-21\right]-5 y_{b 1}+10\left[y_{b 1}-10\right]^{+} } \\
& \left.+10\left[0-y_{b 1}\right]^{+}-\lambda_{b 2}\left[10-\left(y_{b 1}-10\right)\right]-50\right\} .
\end{aligned}
$$

The optimal objective value of the problem above is

$$
v_{b 1}^{L}(21 \mid \lambda)= \begin{cases}11 \lambda_{b 1}-10 \lambda_{b 2}-111 & \text { if }-6 \leq-\lambda_{b 1}+\lambda_{b 2} \leq 14 \\ -\infty & \text { otherwise }\end{cases}
$$

and the optimal solution is 10 when $-6 \leq-\lambda_{b 1}+\lambda_{b 2} \leq 14$ so that $\hat{r}_{b 1}^{\lambda}=10$ when $-6 \leq-\lambda_{b 1}+\lambda_{b 2} \leq 14$.

Using the same argument that we use for the second time period, because we are interested in solving the problem $\max _{\lambda \geq 0}\left\{V_{1}^{L}\left(x_{1} \mid \lambda\right)\right\}$, we only focus on the case where $-6 \leq-\lambda_{a 1}+\lambda_{a 2} \leq 14$ and $-6 \leq-\lambda_{b 1}+\lambda_{b 2} \leq 14$. Noting that the largest value that $\hat{r}_{a 1}^{\lambda}$ can take is 15 , we always have $75 \geq \hat{r}_{a 1}^{\lambda}+\hat{r}_{b 1}^{\lambda}$, and we can assume that $\Delta_{1}^{L}(75 \mid \lambda)=0$ throughout. In this case, by (9) and (45), we have

$$
\begin{aligned}
& v_{\phi 1}^{L}(75 \mid \lambda) \\
& =\min _{y_{\phi 1} \geq 75}\left\{\left[y_{\phi 1}-75\right]+5 y_{\phi 1}+\frac{1}{2} 5\left[y_{\phi 1}-10\right]+\frac{1}{2} 5\left[y_{\phi 1}-25\right]\right\}=662.5 .
\end{aligned}
$$

Because we have $V_{1}^{L}\left(x_{1} \mid \lambda\right)=v_{a 1}^{L}(15 \mid \lambda)+v_{b 1}^{L}(21 \mid \lambda)+v_{\phi 1}^{L}(75 \mid \lambda)$, (46)-(48) imply that

$$
\begin{aligned}
& V_{1}^{L}\left(x_{1} \mid \lambda\right) \\
& \quad=\left\{\begin{array}{cc}
{\left[-2.5 \lambda_{a 2}-50\right]} & \text { if }-6 \leq-\lambda_{a 1}+\lambda_{a 2} \leq 4 \\
+\left[11 \lambda_{b 1}-10 \lambda_{b 2}-111\right]+662.5 & -6 \leq-\lambda_{b 1}+\lambda_{b 2} \leq 14 \\
& -15 \leq \lambda_{a 2} \leq 5,-15 \\
& \leq \lambda_{b 2} \leq 5 \\
{\left[15 \lambda_{a 1}-17.5 \lambda_{a 2}+10\right]} & \text { if } 4 \leq-\lambda_{a 1}+\lambda_{a 2} \leq 14 \\
+\left[11 \lambda_{b 1}-10 \lambda_{b 2}-111\right]+662.5 & -6 \leq-\lambda_{b 1}+\lambda_{b 2} \leq 14 \\
& -15 \leq \lambda_{a 2} \\
& \leq 5,-15 \leq \lambda_{b 2} \leq 5 .
\end{array}\right.
\end{aligned}
$$

Therefore, we can solve the problem $\max _{\lambda \geq 0}\left\{V_{1}^{L}\left(x_{1} \mid \lambda\right)\right\}$ by solving two linear programs and taking the maximum of the optimal objective values obtained from these two linear programs. In this case, it is easy to check that $\max _{\lambda \geq 0}\left\{V_{1}^{L}\left(x_{1} \mid \lambda\right)\right\}=572.5$.

\section{Clark and Scarf's Balance Assumption}

We consider the balance assumption next. By (23), we have

$$
\begin{aligned}
v_{a 2}^{B}\left(x_{a 2}\right)=\min _{y_{a 2} \geq x_{a 2}}\left\{-5 y_{a 2}+10\left[y_{a 2}-10\right]^{+}\right. & \left.+10\left[10-y_{a 2}\right]^{+}\right\} \\
& =5\left[x_{a 2}-10\right]^{+}-50 .
\end{aligned}
$$

The unconstrained minimizer of the objective function of the problem above is 10 so that $\hat{r}_{a 2}=10$. Because the problem parameters for retailer $b$ at the second time period are the same as those for retailer $a, v_{b 2}^{B}\left(x_{b 2}\right)$ has the same form as (50) and we have $\hat{r}_{b 2}=10$. Noting the argument in Case 1 in the proof of Proposition 5 and the fact that $x_{\phi 2}=x_{\phi 1}+q_{\phi 1}-D_{a 1}-D_{b 1} \geq$ $x_{\phi 1}-D_{a 1}-D_{b 1} \geq 50 \geq 20=\hat{r}_{a 2}+\hat{r}_{b 2}$ with probability 1 , we can assume that $\Delta_{2}^{B}\left(x_{\phi 2}\right)=0$ throughout. By (25), we have

$$
v_{\phi 2}^{B}\left(x_{\phi 2}\right)=\min _{y_{\phi 2} \geq x_{\phi 2}}\left\{5 y_{\phi 2}\right\}=5 x_{\phi 2} .
$$

Using (23) and (50), one can check that

$$
\begin{aligned}
& v_{a 1}^{B}(15)=\min _{y_{a 1} \geq 15}\{\left\{y_{a 1}-15\right]-5 y_{a 1} \\
&+\frac{1}{2}\left\{10\left[y_{a 1}-0\right]^{+}+10\left[0-y_{a 1}\right]^{+}+5\left[y_{a 1}-10\right]^{+}-50\right\} \\
&+\frac{1}{2}\left\{10\left[y_{a 1}-15\right]^{+}+10\left[15-y_{a 1}\right]^{+}\right. \\
&\left.\left.+5\left[\left(y_{a 1}-15\right)-10\right]^{+}-50\right\}\right\}=-37.5 \\
& v_{b 1}^{B}(21)=\min _{y_{b 1} \geq 21}\left\{\left[y_{b 1}-21\right]-5 y_{b 1}+10\left[y_{b 1}-10\right]^{+}+10\left[10-y_{b 1}\right]^{+}\right. \\
&\left.+5\left[\left(y_{b 1}-10\right)-10\right]^{+}-50\right\}=-40 .
\end{aligned}
$$

The unconstrained minimizers of the objective functions of the two problems above are respectively 15 and 10 , so that $\hat{r}_{a 1}=15$ and $\hat{r}_{b 1}=10$. Because we have $\hat{r}_{a 1}+\hat{r}_{b 1}=25 \leq 75=x_{\phi 1}$, we have $\Delta_{1}^{B}\left(x_{\phi 1}\right)=0$ by the argument in Case 1 of Proposition 5. Finally, by (25) and (51), we have

$$
\begin{aligned}
& v_{\phi 1}^{B}(75) \\
& =\min _{y_{\phi 1} \geq 75}\left\{\left[y_{\phi 1}-75\right]+5 y_{\phi 1}+\frac{1}{2} 5\left[y_{\phi 1}-10\right]+\frac{1}{2} 5\left[y_{\phi 1}-25\right]\right\}=662.5 .
\end{aligned}
$$


Therefore, we obtain $V_{1}^{B}\left(x_{1}\right)=v_{a 1}^{B}(15)+v_{b 1}^{B}(21)+v_{\phi 1}^{B}(75)=585>$ $572.5=\max _{\lambda \geq 0}\left\{V_{1}^{L}\left(x_{1} \mid \lambda\right)\right\}$.

We also note that $V_{1}^{L}\left(x_{1} \mid 0\right)=501.5<585=V_{1}^{B}\left(x_{1}\right)$ by (49). Therefore, the lower bound computed under the relaxation strategy of Federgruen and Zipkin [14] is different from that computed under the balance assumption.

It turns out that the balance assumption is satisfied for this problem instance, which implies that the lower bound computed under the balance assumption is tight, and we have $V_{1}^{B}\left(x_{1}\right)=V_{1}\left(x_{1}\right)$ by Proposition 5. To see this, we first note that we can assume without loss of generality that $x_{a 2} \leq 25$, because the total demand at retailer $a$ does not exceed 25 with probability 1 , and we would keep the excess inventory at the warehouse rather than at the retailer. Similarly, we can assume without loss of generality that $x_{b 2} \leq 20$. We also have $x_{\phi 2}=x_{\phi 1}+q_{\phi 1}-D_{a 1}-D_{b 1} \geq$ $x_{\phi 1}-D_{a 1}-D_{b 1} \geq 50 \geq 20=\hat{r}_{a 2}+\hat{r}_{b 2}$ with probability 1 . If we solve problem (1)-(3) for the second time period without the constraint $y_{a 2}+y_{b 2} \leq x_{\phi 2}$, then one can check that the optimal solution is $y_{a 2}=\max \left\{x_{a 2}, 10\right\}, y_{b 2}=\max \left\{x_{b 2}, 10\right\}$, and $y_{\phi 2}=x_{\phi 2}$. Therefore, because we have $\max \left\{x_{a 2}, 10\right\}+\max \left\{x_{b 2}, 10\right\} \leq 25+20 \leq 50 \leq x_{\phi 2}$, the constraint $y_{a 2}+y_{b 2} \leq x_{\phi 2}$ is redundant in problem (1)-(3). Because we have $x_{\phi 2} \geq \hat{r}_{a 2}+\hat{r}_{b 2}$ and the constraint $y_{a 2}+y_{b 2} \leq x_{\phi 2}$ is redundant in problem (1)-(3), the balance assumption is satisfied for the second time period. Similarly, if we solve problem (1)-(3) for the first time period without the constraint $y_{a 1}+y_{b 1} \leq x_{\phi 1}$, then one can check that the optimal solution is $y_{a 1}=15, y_{b 1}=21$, and $y_{\phi 1}=75$. Therefore, because we have $15+21 \leq 75=x_{\phi 1}$, the constraint $y_{a 1}+y_{b 1} \leq x_{\phi 1}$ is redundant in problem (1)-(3). Because we have $x_{\phi 1}=75 \geq 25=\hat{r}_{a 1}+\hat{r}_{b 1}$ and the constraint $y_{a 1}+y_{b 1} \leq x_{\phi 1}$ is redundant in problem (1)-(3), the balance assumption is satisfied for the first time period. On the other hand, the inventory position at retailer $a$ at the beginning of the second time period depends on the realization of the demand at the first time period. This implies that the trajectory of the state variable $\left\{x_{t}: t \in \mathcal{T}\right\}$ depends on the sample path, and the lower bound obtained by the Lagrangian relaxation strategy is not necessarily tight.

\section{ACKNOWLEDGMENTS}

The authors thank two anonymous referees for their useful comments that strengthened the exposition and the computational results. This work was supported in part by National Science Foundation grant DMI-0422133.

\section{REFERENCES}

[1] D. Adelman and A.J. Mersereau, Relaxations of weakly coupled stochastic dynamic programs, Oper Res (in press).

[2] S. Axsater, "Supply chain operations: Serial and distribution inventory systems," Handbook of operations research, supply chain management: Design, coordination and operation, S.C. Graves and A.G. de Kok (Editors), Elsevier Science, Amsterdam, North Holland, 2003, pp. 525-559.

[3] S. Axsater, J. Marklund, and E.A. Silver, Heuristic methods for centralized control of one-warehouse, n-retailer inventory systems, Manufact Serv Oper Management 4 (2002), 75-97.

[4] M.S. Bazaraa, H.D. Sherali, and C.M. Shetty, Nonlinear programming: Theory and algorithms, 2nd ed., Wiley, New York, 1993.

[5] D.P. Bertsekas, Nonlinear programming, Athena Scientific, Belmont, MA, 1995.

[6] D.P. Bertsekas, Dynamic programming and optimal control, Athena Scientific, Belmont, MA, 2001.
[7] R.K. Cheung and W.B. Powell, An Algorithm for multistage dynamic networks with random arc capacities, with an application to dynamic fleet management, Oper Res 44 (1996), 951-963.

[8] A.J. Clark and H. Scarf, Optimal policies for a multi-echelon inventory problem, Management Sci 64 (1960), 475-490.

[9] M.K. Dogru, Optimal control of one-warehouse multi-retailer systems: An assessment of the balance assumption, $\mathrm{PhD}$ Thesis, Technische Universiteit Eindhoven, Eindhoven, The Netherlands, 2005.

[10] M.K. Dogru, A.G. de Kok, and G.J. van Houtum, A numerical study on the effect of the balance assumption in one-warehouse multi-retailer inventory systems, Beta working paper, WP 135, Department of Technology Management, Technische Universiteit Eindhoven, 2005.

[11] G.L. Eppen and L. Schrage, "Centralized ordering policies in a multi-warehouse system with leadtimes and random demand," Multi-level production/inventory control systems: Theory and practice, L. Schwarz (Editor), North Holland, Amsterdam, The Netherlands, 1981, pp. 51-69.

[12] A. Federgruen, "Centralized planning models for multiechelon inventory systems under uncertainty," Handbook in operations research and management science, volume on logistics of production and inventory, S.C. Graves, A. H. G.R. Kan, and P.H. Zipkin (Editors), North Holland, Amsterdam, 1993, pp. 3-50.

[13] A. Federgruen and P. Zipkin, Allocation policies and cost approximations for multilocation inventory systems, Naval Res Logist 31 (1984a), 97-129.

[14] A. Federgruen and P. Zipkin, Approximations of dynamic, multilocation production and inventory problems, Management Sci 30 (1984b), 69-84.

[15] A. Federgruen and P. Zipkin, Computational issues in an infinite horizon multi-echelon inventory model, Oper Res 32 (1984c), 818-836.

[16] B. Fox, Discrete optimization via marginal analysis, Management Sci 13 (1966), 210-216.

[17] G. Gallego, O. Ozer, and P. Zipkin, Bounds, heuristics, and approximations for distribution systems, Oper Res 55 (2007), 503-517.

[18] P.L. Jackson, Stock allocation in a two-echelon distribution system or "what to do until your ship comes in", Management Sci 34 (1988), 880-895.

[19] U.S. Karmarkar, The multiperiod multilocation inventory problem, Oper Res 29 (1981), 215-228.

[20] U.S. Karmarkar, The multilocation multiperiod inventory problem: Bounds and approximations, Management Sci 33 (1987), 86-94.

[21] H. Topaloglu, Using Lagrangian relaxation to compute capacity-dependent bid-prices in network revenue management, Cornell University, School of Operations Research and Information Engineering, 2006, Available at http://legacy.orie. cornell.edu/ huseyin/publications/publications.html.

[22] H. Topaloglu and S. Kunnumkal, Approximate dynamic programming methods for an inventory allocation problem under uncertainty, Naval Res Logist Quart 53 (2006), 822-841.

[23] P.P. Varaiya, Lecture notes on optimization, Unpublished manuscript, University of California, Department of Electrical Engineering and Computer Science, 1998, Available at http:// paleale.eecs.berkeley.edu/ varaiya.

[24] L.A. Wolsey, Integer Programming, Wiley, New York, 1998. 